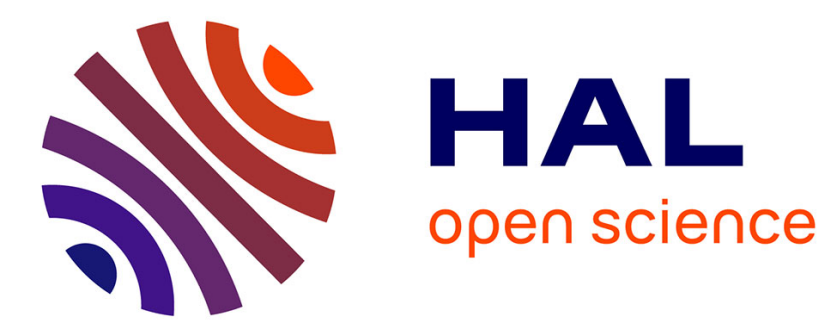

\title{
Support vector regression-based 3D-wavelet texture learning for hyperspectral image compression
}

Nadia Zikiou, Mourad Lahdir, David Helbert

\section{To cite this version:}

Nadia Zikiou, Mourad Lahdir, David Helbert. Support vector regression-based 3D-wavelet texture learning for hyperspectral image compression. The Visual Computer, 2020, 36, pp.1473-1490. 10.1007/s00371-019-01753-z . hal-02279113

\section{HAL Id: hal-02279113 \\ https://hal.science/hal-02279113}

Submitted on 11 Dec 2019

HAL is a multi-disciplinary open access archive for the deposit and dissemination of scientific research documents, whether they are published or not. The documents may come from teaching and research institutions in France or abroad, or from public or private research centers.
L'archive ouverte pluridisciplinaire HAL, est destinée au dépôt et à la diffusion de documents scientifiques de niveau recherche, publiés ou non, émanant des établissements d'enseignement et de recherche français ou étrangers, des laboratoires publics ou privés. 


\title{
Support Vector Regression based 3D-Wavelet Texture Learning for Hyperspectral Image Compression
}

\author{
Nadia. Zikiou • Mourad. Lahdir · David. Helbert
}

June 18, 2019

\begin{abstract}
Hyperspectral imaging is known for its rich spatial-spectral information. The spectral bands provide the ability to distinguish substances spectra which is substantial for analyzing materials. However, highdimensional data volume of hyperspectal images is problematic for data storage. In this paper, we present a lossy hyperspectral image compression system based on the regression of 3D wavelet coefficients. The 3D wavelet transform is applied to sparsely represent the hyperspectral images (HSI). A support vector machine regression (SVR) is then applied on wavelet details and provides vector supports and weights which represent wavelet texture features. To achieve the best possible overall rate-distortion performance after regression, entropy encoding based on run-length encoding (RLE) and arithmetic encoding are used. To preserve the spatial pertinent information of the image, the lowest subband wavelet coefficients are furthermore encoded by a lossless coding with differential pulse code modulation (DPCM). Spectral and spatial redundancies are thus substantially reduced. Experimental tests are performed over several hyperspectral images from airborne and spaceborne sensors and compared with the main existing algorithms. The obtained results show that the proposed compression method has high performances in terms of rate-distortion and spectral fidelity. Indeed, high PSNRs and classification accuracies, which could exceed $40.65 \mathrm{~dB}$ and $75.8 \%$ respectively, are observed for all decoded HSI images and overpass those given by
\end{abstract}

N. Zikiou · M.Lahdir

Laboratory of Analysis and Modeling of Random Phenomena (LAMPA),UMMTO, BP 17 RP, 15000, Tizi-Ouzou Algeria

E-mail: zikiounadia@gmail.com

D. Helbert

Xlim CNRS University of Poitiers, 11 Bd Marie et Pierre Curie, BP 30179, 86962, Chasseneuil Cedex France many cited famous methods. In addition, the evaluation of detection and compression over various bands shows that spectral information is preserved using our compression method.

Keywords Remote sensing - Hyperspectral image · Lossless compression · Image coding · Spectral classification - Texture feature learning

\section{Introduction}

Nowadays images have an important role in many fields, notably in remote sensing applications like forestry, city planning and land use classification in agriculture, etc. Hyperspectral imaging has become a popular remote sensing as it achieves hundreds of contiguous spectral bands which provide the ability to distinguish virtually the major part of materials. The aim of spectral analysis is to find objects, identify materials or detect processes. The principle is to compare waveforms of image spectrum to those of known substance spectra, and to precisely discriminate different types using the adequate spectral information. Hyperspectral imaging sensors provide high quality data for utility assessments due to their advanced technologies. AVIRIS is an airborne hyperspectral sensor that records 224 contiguous bands, from $400 \mathrm{~nm}$ to $2500 \mathrm{~nm}$, of size 2048 rows by 614 columns and 2 bytes/sample per band (over 537 Megabytes per image). HYDICE is also an airborne sensor with high spectral resolution; it provides $210 \mathrm{spec}-$ tral bands from 400 to $2500 \mathrm{~nm}$ and $10 \mathrm{~nm}$ wide covering the spectral range. HYPERION is an spaceborne hyperspectral sensor, it is a pushbroom imaging instrument. It records 220 spectral bands from $400 \mathrm{~nm}$ to $2500 \mathrm{~nm}$ with a $6.5 \mathrm{Km}$ swath width and a spatial resolution of $30 \mathrm{~m}$. Due to such highly correlated data and 
high data volumes, the hyperspectral data compression has become increasingly important in data processing, analysis and storage. Huge volumes of daily-generated data can be handled and stored only with the help of efficient compression algorithms. Image compression techniques fall broadly into two main categories: lossless and lossy compression depending on whether the original image can be precisely re-generated from the compressed data. Although the lossless compression is often used to preserve the full information of original images, it provides a lower compression ratio than that of lossy compression, which is a disadvantage for channel transmission and storage. For the last few years, discrete wavelet transform (DWT) has been commonly used for image compression and restoration [1-4]. It is a robust technique for image compression due to its multiresolution feature, scalability and flexibility. Many promising 3D image compression algorithms based on wavelet transforms were recently proposed [5-14]. In [15-17] authors suggest different techniques for 2D image compression method based on combining DWT and support vector machine (SVM). They remove blocking artifacts and improve the quality of the $2 \mathrm{D}$ image. The wavelet transform generates approximation coefficients (AC) and detail coefficients (DC). Approximation coefficients represent pixel values of an image dataset while detail coefficients are distributed in horizontal, vertical and diagonal sub-bands. Moreover, the wavelet transform provides fundamental trade-off between frequency and time localization. Although these methods are very effective for 2D grey-level and color image compression, many papers as $[18,19]$ highlight that most of these coding methods do not consider the spectral characteristics of hyperspectral images (HSI). This may be problematic for the identification of classes and objects in 3D HSI and could notably affect analysis results. $[18,19]$ present an HSI compression algorithm based on the three-order tensor which can simultaneously consider both the spatial and spectral domains of the data cube. However the spectral correlation (band redundancy) is generally considered stronger than spatial correlation. An effective lossless compression algorithm based on classification is proposed in [20], it is based on the selection of bands with considerable information and performed with K-means to obtain classification map. The introduction of a multilinear regression model on this algorithm allowed to construct the highquality side information of each class. In [21], authors proposed hyperspectral lossless coding scheme based regression wavelet analysis. The proposed scheme allowed to increase the wavelet coefficients independence and so reduce redundancy. According to the results, this approach yields to better performances than many other famous techniques such as PCA. In this paper, an effective hyperspectral image compression method is proposed. It considers the spectral information while preserving the most pertinent information in the image. Our method is based on the $3 \mathrm{D}$ wavelet transform and support vector regression (SVR). The regression is applied on spectral dimension data to provide the most important coefficients in the sequence, that are called support vectors (SVs). This method allows the most significant part of spectral to be preserved. First of all, the HSI is sparsely represented by DWT to obtain $3 \mathrm{D}$ wavelet coefficients which define the $\mathrm{AC}$ and DC. The compression step is then performed by application of differential pulse code modulation (DPCM) on approximate coefficients and this generates the first code sequence. Note that this code sequence includes a large amount of image energy, which is efficient for image reconstruction. While the SVR is used to produce the vector and its weight support of spectral wavelet detail coefficients, the obtained coefficients are encoded by run-length encoding (RLE) and arithmetic encoding and the other code sequence is generated. The two code sequences are then used for HSI reconstruction. This approach eliminates the spatial and spectral redundancies which are observed in HSI. Compared to several other methods, the approach considerably preserves the quality of the spectral HSI data. The classification performances of the method have been also evaluated. Results show that the proposed method yields to high classification accuracy and overcomes famous other compression/classification methods. This paper is organized as follows. The wavelet-SVM compression algorithm for hyperspectral images is proposed in section 2. Section 3 shows the experimental results obtained from several HSI and the comparisons with the main existing algorithms. Section 4 gives the classification performances of the method. Section 5 describes the impact of the proposed method on the spectral information of HSI. Section 6 draws some conclusions.

\section{Compression Algorithm}

\subsection{Principle}

First of all the proposed HSI compression provides wavelet coefficients by applying the 3D DWT transform to get AC and DC (see Fig. 1). We perform three level wavelets decomposition to arrive time computation and quality. The approximation signal displays pixel values and the detail signal displays the horizontal, vertical, and diagonal details of an image dataset. These different coefficients will be processed according to their importance. An adapter quantization, named deadzone, is used on 


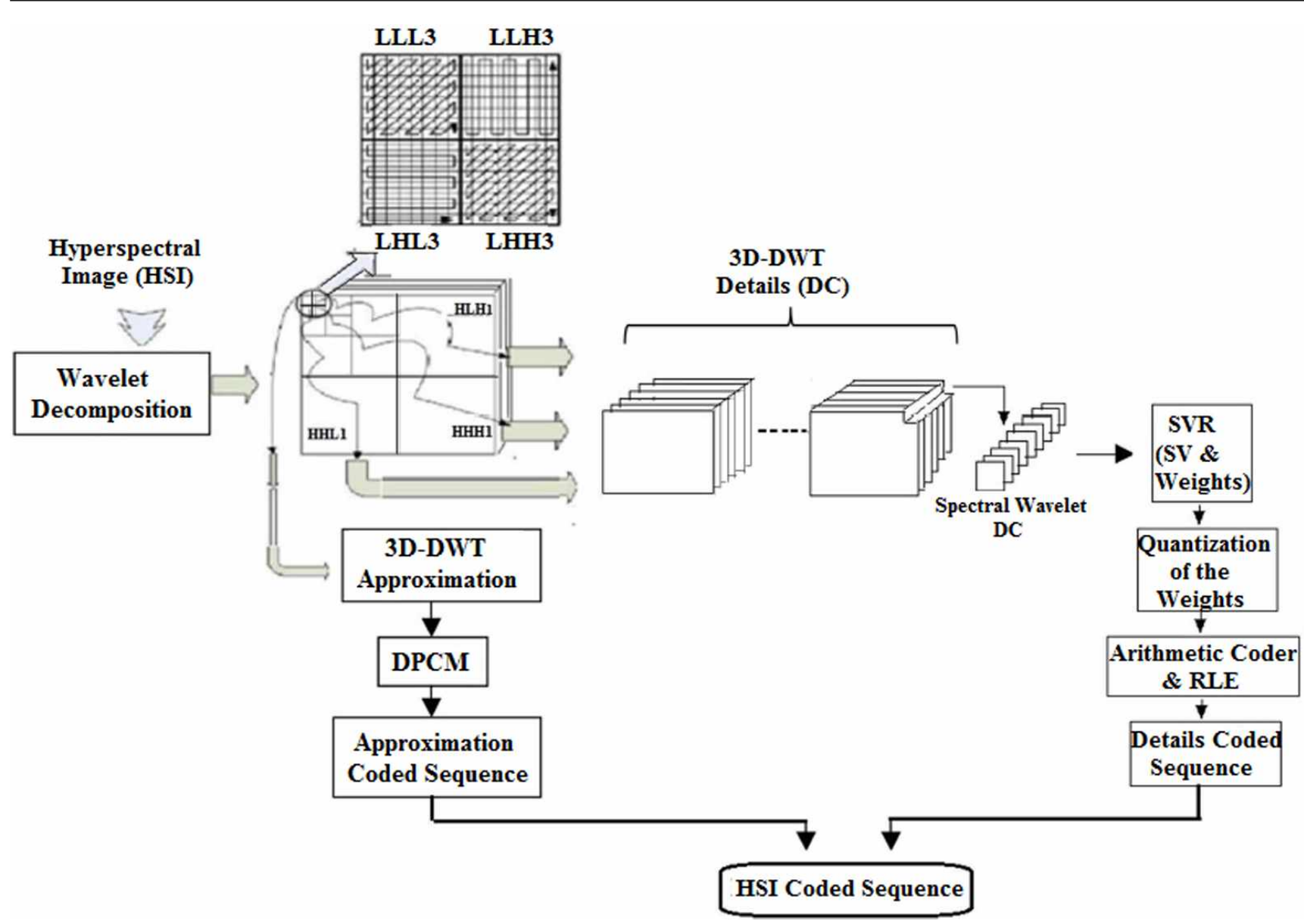

Fig. 1: The Wavelet-SVR compression algorithm.

these coefficients and enables all source values which are considered low to be quantified in a single same value (usually zero). The lowest sub-band wavelet coefficients are encoded by differential pulse code modulation (DPCM) to preserve the large amount of image energy present in this sub-band. The SVM regression is thus applied to the spectral wavelet detail sub-bands coefficients to represent the original data and eliminate redundancy. Weights and support vectors are computed and encoded together by entropy encoder. The aim of this procedure is to preserve, as well as possible, the spectral information of HSI data which is substantially important for the subsequent 3D HSI data analysis. Furthermore the spatial information is preserved using DPCM on low-frequency image.

\subsection{Wavelet transform}

The wavelet transform is an effective mathematical tool for time-frequency analysis in signal processing and pattern recognition. A wavelet is a normalized function $\psi \in L^{2}(\mathbb{R})$ with a null mean [22]. A discrete wavelet is the inner product of finite-length sequence and a discretized wavelet basis with a scale factor $s_{0}^{a}$ and a translation factor $b$ :

$\psi_{b, a}(n)=\frac{1}{\sqrt{s_{0}^{a}}} \psi\left(\frac{n-b}{s_{0}^{a}}\right)$.

The wavelet coefficients $W_{f}(b, a)$ of a sequence $f_{(n)}$ is obtained by projecting $f$ onto the family $\psi_{b, a}(n)$ :

$W_{f}(b, a)=<f(t), \psi_{b, a}^{*}>=\sum_{n=0}^{N} f(n) \cdot \psi_{b, a}^{*}(n)$,

where $\psi_{b, a}^{*}$ denotes the conjugate of $\psi_{b, a}$ and $<$. $>$ the scalar product. Parameter $a$ determines the frequency region. A large $|a|$ indicates low frequencies, while a small $|a|$ indicates high frequencies [23]. Note that for the dyadic discrete wavelet transform $s_{0}=2$.

In image processing, a 2D decomposition algorithm to compute discrete wavelet transform consists in choosing a low pass and a high pass filter, known as analysis filter pair [24]. The analysis filter divides data into low frequency and the high frequency components. The data can be sub-sampled by two. For a hyperspectral 
image, 1D transforms in the third direction are added. In a 3D transform, 8 subband cubes are created at the first decomposition level. The low pass sub-band at the top of the pyramid contains approximate coefficients, which are the most significant information of the image. The high frequency sub-bands contain the edges of the low resolution image. The size of high frequency components of DWT is furthermore increased by $3 \mathrm{D}$ bicubic interpolation with factor 2. Low frequency (A) and high frequency (D) wavelet coefficients of an image in resolution level $l$ in the three dimensions $(x, y, z)$ are defined as follows:

$A_{n}^{(l)}(x, y, z)=\frac{1}{2}\left(A_{2 n}^{(l-1)}+A_{2 n+1}^{(l-1)}\right)$

$D_{n}^{(l)}(x, y, z)=\frac{1}{2}\left(A_{2 n}^{(l-1)}-A_{2 n+1}^{(l-1)}\right)$

Wavelet transform codings provide many interesting advantages over other transform methods. They have been used extensively in such research work as, EZW [25], SPIHT [26], SPECK [27, 28] and EBCOT [29] that are all wavelet based schemes. In this study, we use the Cohen-Daubechies-Feauveau (CDF) 9/7 tap biorthogonal filters [30]. This transform is one of the most popular transforms used in image compression [26-29, 31], it produces floating point wavelet coefficients. Filters CDF 5/3, Db4 and Symlet Wavelets filters [32, 33] are also tested to prove the effect of the approach on the results.

\subsection{Differential Pulse Code Modulation}

The lowest sub-band of the HSI is encoded by DPCM for including a large amount of image energy. It is based on the fact that most source signals show significant correlation between successive samples so the encoding uses the redundancy in sample values, which implies a lower bit rate. A linear prediction is employed to predict the coefficients, by minimizing the expected value of the squared error inside each band. The linear approximate wavelet coefficient prediction function is:

$\widehat{A}_{x, y, z}=\sum_{i=1}^{M_{z}}\left(p_{z, s, i} A_{x, y, z}+p_{z, s, 0}\right)$.

where $A_{x, y, z}$ and, $\widehat{A}_{x, y, z}$ represent the pixel AC subband value and predictive value at band $z$ with the spatial location $(x, y)$ respectively, $M_{z}$ is the prediction length that is used in the prediction, and $p_{z, s, i}$ denotes the prediction coefficients for cluster $s$ at band $z$.

\subsection{Support Vector Machine Regression For Image} Compression

\subsubsection{Principle of SVM regression}

As a recent machine learning method, SVM has been commonly used in many areas because of its good generalization capability. Although SVM was initially designed to solve classification problems the Support vector $(\mathrm{SV})$ techniques can be successfully applied in regression, i.e., for functional approximation problems. The application of SVM learning to regression problems is used in this paper. In regression, the learning machine is given a set of training points. The real function is approximated within a predefined error or margin of tolerance, by choosing the lowest number of training points termed Support Vectors. For each Support Vector, there is a corresponding weight (W) [34].

Let the set of training data be

$\mathcal{C}=\left\{\left(\mathbf{x}_{1}, \mathbf{y}_{1}\right), \ldots,\left(\mathbf{x}_{N}, \mathbf{y}_{N}\right)\right\}$

where $\mathbf{x}_{i}(1 \leq i \leq N)$ is a vector in the input space, which represents in our case the spectral wavelet vector for each coefficient of DC sub-band and, $\mathbf{y}_{i} \in \mathbb{R}$ the corresponding target. The number of training samples is equal to $\mathrm{N}$. To predict data $\mathbf{x}$, we want to learn the following regression function $g(\mathbf{x})$ :

$g(\mathbf{x})=<w, \phi(\mathbf{x})>+B$,

where $w \in \mathbb{R}^{N}$ is the weight vector in the kernel feature space, $\phi(\mathbf{x})$ is the kernel feature map of data $\mathbf{x}$, and $b \in \mathbb{R}$ is a bias. The solution is obtained by reformulating and solving a convex optimization problem proposed by Vapnik [35] . The final prediction model can be formulated as follows:

$g(\mathbf{x})=\sum_{i=1}^{N}\left(\alpha_{i}-\alpha_{i}^{*}\right) k\left(\mathbf{x}_{i}, \mathbf{x}\right)+B$.

where $k(.,$.$) is a kernel function, coefficients \alpha_{i}$ and $\alpha_{i}^{*}$ are the SV support. In data compression, the smaller number of support vectors allows a higher compression ratio.

\subsubsection{Definition of kernel function}

The Support Vector machine is a dot product kernel. The kernel function is to map inputs in the low-dimensional space into a high-dimensional feature space. The target data can then be classified by using the hyperplane. SVM kernel function $k(\mathbf{x})$ must satisfy the Mercer's conditions [36]. In theory, the most well known SVM kernels are polynomial, Gaussian, linear, sigmoidal or a radial basis function. The polynomial kernel and the Gaussian radian basis function are defined as follows: 
- A polynomial kernel:

$$
k\left(\mathbf{x}_{\mathbf{i}}, \mathbf{x}_{\mathbf{j}}\right)=\left[<\mathbf{x}_{\mathbf{i}}, \mathbf{x}_{\mathbf{j}}>+c\right]^{q},
$$

where $q$ is the degree of the polynomial function and $c \in[0,+\infty[$. To note that a linear kernel can be defined with $q=1$.

- Gaussian radial basis function:

$$
k\left(\mathbf{x}_{\mathbf{i}}, \mathbf{x}_{\mathbf{j}}\right)=e^{-\gamma\left\|\mathbf{x}_{\mathbf{i}}-\mathbf{x}_{\mathbf{j}}\right\|^{2}},
$$

with $\gamma$ a free parameter. We have chosen to use $\epsilon$-SVR model with three SVM kernels (linear, polynomial and Gaussian), which is the original SVM formulation for Regression (SVR). It uses parameter $\epsilon \in[0,+\infty[$ to apply a penalty to the optimization for points which were not correctly predicted.

\subsubsection{Quantization and arithmetic coding}

The nonzero weight coefficients are not integers so they should be quantized before the arithmetic coding. We use a floating-point quantizer for this purpose. To reduce the physical size of the quantized sequence, we apply the RLE coding. It replaces sequences of the same data values within a file by a count number and a single value. To achieve significant compression, the classical arithmetic coder is used [37]. It transforms the sequence of quantizied data to a bitstream by transmitting the most probable weight coefficients in fewer bits. The bitstream of each HSI sub-band for the three wavelet decomposition levels is saved. The detail coding sequence of the HSI dataset is obtained by taking all the bitstreams.

After SVM regression, weight and support vectors are encoded together by entropy coding. The main steps of the encoding algorithm are given briefly in algorithm 1. The decompression of the proposed SVR-3D Wavelet method mainly contains the reconstruction process. It is briefly shown in Algorithm. 2.

\section{Experimental Results}

To demonstrate the effectiveness of the proposed 3DDWT + SVR method, An extensive amount of experiments have been performed over calibrated and uncalibrated hyperspectral images acquired by AVIRIS, HYDICE and HYPERION sensors. In this section, obtained results are shown and discussed.

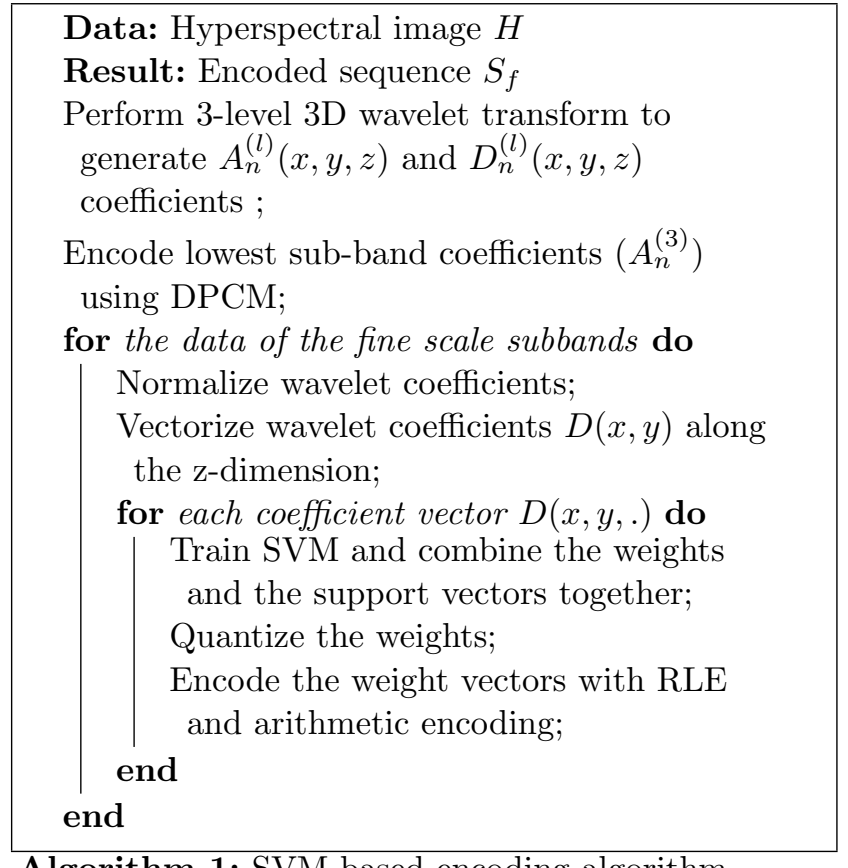

Algorithm 1: SVM-based encoding algorithm

\author{
Data: Encoded sequence $S_{f}$ \\ Result: Reconstructed Hyperspectral image $H$ \\ Decode the bitstream by RLE and arithmetic \\ decoding; \\ Perform the high scale subbands ; \\ Inverse normalize of fine scale subbands \\ coefficients; \\ Decode lowest sub-band coefficients using the \\ inverse DPCM; \\ Reconstruct HSI with inverse wavelet \\ transform;
}

\section{Algorithm 2: Decompression}

\subsection{Experimental Data Set}

We consider four airborne hyperspectral images : the Yellowstone uncalibrated scene 0, Cuprite scene 1 and Indiana Pines images acquired by Airborne Visible Infrared Imaging Spectrometer (AVIRIS) sensor, and the Washington DC Image acquired by Hyperspectral Digital Image Collection Experiment (HYDICE) sensor. To estimate the robustness of our method, an spaceborne hyperspectral remote sensing data system is also considered. Thus, two other hyperspectral images acquired by HYPERION sensor are considered. i.e. Mt. St. Helens and Erta Ale images. The choice of these images is due to the variety of information which is useful for analyzing the effect of SVM learning on different datasets (water, agglomeration, ground, ...). The detailed de- 
scriptions of these HSIs datasets are shown in Fig. 2 and Fig. 3.

Yellowstone uncalibrated scene 0 image The image was acquired in 2006. It contains 224 spectral bands with 512 by 614 -pixel resolution for each band and 16 bits per pixel per band (bpppb). This AVIRIS image can be downloaded from NASA web site ${ }^{1}$. Fig. 2 (a) shows the $71^{\text {st }}$ band of Yellowstone image.

Cuprite image The Cuprite image was acquired in 1996, it is included in standard 1997 AVIRIS calibrated radiance images. This image is a $20 \mathrm{~m}$ spatial resolution containing 224 spectral bands between 0.4 and $2.5 \mu \mathrm{m}$. The noisy bands $(1,2,109-112,156-167)$ of Cuprite data are removed in the experiment. Fig. 2 (b) shows the $2^{\text {nd }}$ of Cuprite image.

Indiana Pines image The HSI was acquired over the agricultural Indiana Pines test site in Northwestern Indiana in 1992. It contains 220 bands across the spectral range from 0.4 to $2.5 \mu \mathrm{m}$ with 145 by 145 pixels for each band. This HSI dataset can be downloaded from the web site $^{2}$. We removed noisy bands in the experiment i.e. 2,3, 104-108, 150-165, 219 and 220. The fourth band of this image is shown on Fig. 2 (c).

Washington DC Image The HSI was acquired over the Washington DC Mall, it can be downloaded from the web site ${ }^{3}$. It consists of 191 spectral bands from 0.4 to $2.4 \mathrm{~nm}$ and of 1280 by 307 spatial size. The $10^{\text {th }}$ band of this image is shown on Fig. 2 (d).

Mt. St. Helens This HSI image was acquired in 2009 by HYPERION sensor and provided by the EO-1 Mission, NASA/USGS. This image is a $30 \mathrm{~m}$ spatial resolution containing 242 spectral bands with $3242 \times 256$ pixel resolution for each band and $12 \mathrm{bpppb}$. This image can be downloaded from ${ }^{4}$. The image is shown on Fig. 3 (a).

Erta Ale Image The Erta Ale Image was acquired in 2010 by HYPERION sensor. As Mt. St. Helens image, The Erta Ale is a $30 \mathrm{~m}$ spatial resolution with $242 \mathrm{spec}-$ tral bands and 12 bpppb. Each band is a $3187 \times 256$

\footnotetext{
1 http://aviris.jpl.nasa.gov/html/aviris.freedata. html

2 http://www.ehu.eus/ccwintco/uploads/2/22/Indian_ pines.mat

3 http://cobweb.ecn.purdue.edu/ biehl/ Hyperspectral_Project.zip

4 https://coding.jpl.nasa.gov/hyperspectral/
}

pixel resolution. The image can be downloaded from ${ }^{5}$ ( Fig. 3 (b)).

For our experiments, we use different size data cubes. The sizes of the test sub-images are $512 \times 512$ for Yellowstone, $256 \times 256$ for Cuprite, $128 \times 128$ for Indiana Pines and $256 \times 256$ for Washington DC image as shown on Fig. 2. For both the Mt. St. Helens and the Erta Ale images, a size of $768 \times 256$ is used (Fig. 3).

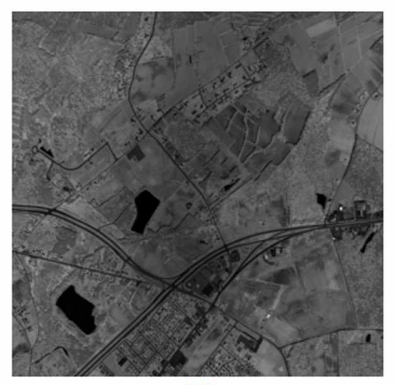

(a)

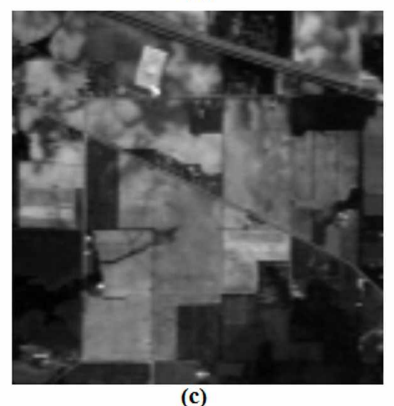

(c)

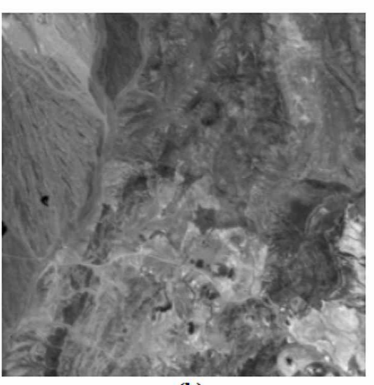

(b)

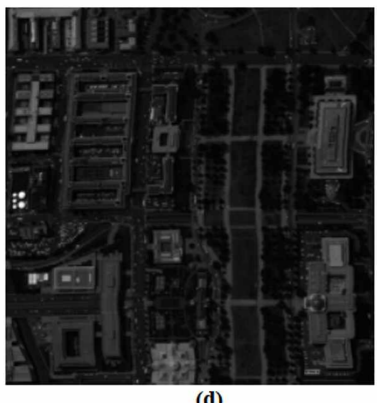

(d)
Fig. 2: Airborne compression dataset: (a) Yellowstone scene 0 image $(512 \times 512)$; (b) Cuprite image $(256 \mathrm{x}$ $256),($ c) Indiana Pines image $(128 \times 128)$ and $(d)$ Washington DC image $(256 \times 256)$.

\subsection{Evaluation method}

Our algorithm is implemented with MATLAB and we have used LibSVM ${ }^{6}$ for $\epsilon$-SVR. To evaluate the performance, following measurements are adopted as criteria to measure the reconstruction error:

- Bit-rate quantified using bit-per-pixel (bpp):

Bit rate $=\frac{\text { Number of bits per pixel in the original image }}{\text { CR }}$,

\footnotetext{
5 https://coding.jpl.nasa.gov/hyperspectral/

6 http://www.csie.ntu.edu.tw/ cjlin/libsvm/.
} 


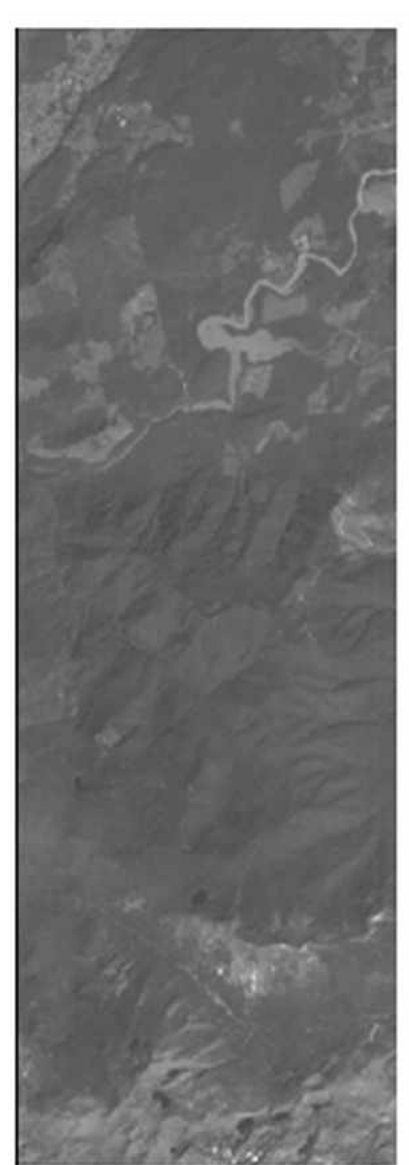

(a)

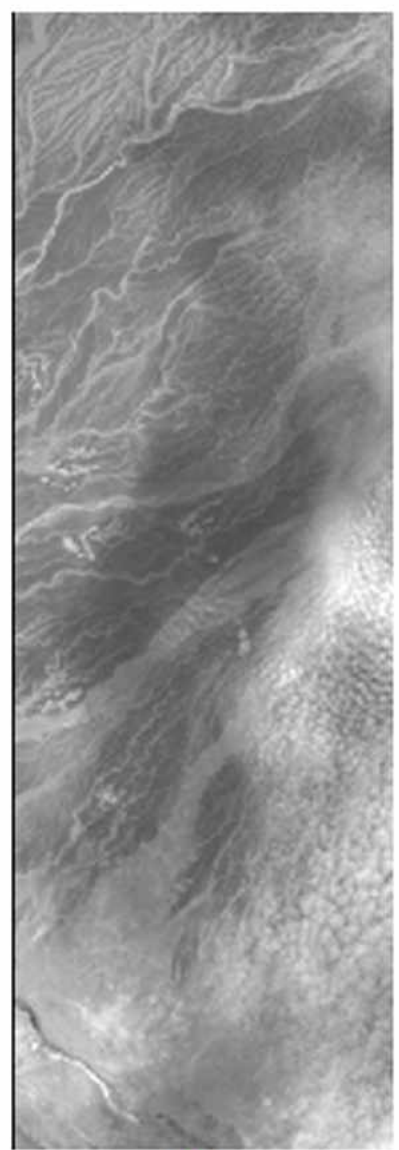

(b)
Fig. 3: Spaceborne compression dataset: (a) Mt. St. Helens image $(768 \times 256)$; (b) Erta Ale image $(768 \times 256)$.

where CR is the Compression Ratio which is formulated as follows:

$\mathrm{CR}=\frac{\text { Original image size in bits }}{\text { Compressed bitstream size in bits }}$

- Rate Distortion Performance (SNR):

$\mathrm{SNR}=10 \log _{10} \frac{\|H\|^{2}}{\|H-\widehat{H} \mid\|^{2}}$

where $\mathrm{H}$ is the original image and $\widehat{H}$ is the reconstructed image.

- Mean-square-error (MSE):

$\operatorname{MSE}(\mathbf{u}, \mathbf{v})=\frac{1}{M} \sum_{u, v}\|\mathbf{u}-\mathbf{v}\|^{2}$

- Peak signal-to-noise ratio (PSNR):

$\operatorname{PSNR}(\mathbf{u}, \mathbf{v})=10 \log _{10} \frac{\left(2^{r}-1\right)^{2}}{\operatorname{MSE}(\mathbf{u}, \mathbf{v})}$
- Structural similarity (SSIM) index [38, 39]:

$\operatorname{SSIM}(\mathbf{u}, \mathbf{v})=\frac{\left(2 \mu_{\mathbf{u}} \mu_{\mathbf{v}}+c_{1}\right)\left(2 \sigma_{\mathbf{u v}}+c_{2}\right)}{\left(\mu_{\mathbf{u}}^{2}+\mu_{\mathbf{v}}^{2}+c_{1}\right)\left(\sigma_{\mathbf{u}}^{2}+\sigma_{\mathbf{v}}^{2}+c_{2}\right)}$

where $\mathbf{u}$ and $\mathbf{v}$ denote the vectors of original images of size $M, \mu_{\mathbf{u}}$ and $\mu_{\mathbf{v}}$ the mean of $\mathbf{u}$ and $\mathbf{v}, \sigma_{\mathbf{u}}$ and $\sigma_{\mathbf{v}}$ the standard deviation of $\mathbf{u}$ and $\mathbf{v} \cdot c_{1}=$ $\left(k_{1} L\right)^{2}$ and $c_{2}=\left(k_{2} L\right)^{2}$ are two variables to avoid instability with weak denominator, $L=2^{r}-1$ is the dynamic range of the pixel-values and $r$ denotes the maximum bit depth. $k_{1}$ and $k_{2}$ are set to 0.01 and $0.03[38,39]$.

- McNemar's test: To evaluate the statistical difference between two classifiers, the McNemar's test is performed. This standardized statistical test describes if two classifiers are significantly different. The $Z$ value of McNemar's test is given as in [40]:

$Z=\frac{f r_{12}-f r_{21}}{\sqrt{f r_{12}-f r_{21}}}$

where $f r_{12}$ represents the correctly classified samples by the first classifier and incorrectly classified samples by the second one. According to state of the art, the value of $Z$ which reflects the significant difference of one classifier from another is estimated between 1.96 [40] and 2.58 [41].

\subsection{Discussions on kernels}

To evaluate the impact of kernels on the compression, the compression scheme is tested over the six datasets with Gaussian (G), polynomial (P) and linear (L) kernels. To fix the optimal SVR parameters, we empirically fix values which maximize the estimated PSNRs. For the polynomial kernel, degree $q$ is equal to 0.6 and offset $\mathrm{c}$ is equal to 0.1 . For the Gaussian kernel, $\gamma$ is set to 0.9129 .

The visual results for one sub-band of reconstructed images for each dataset are shown in Fig. 4 for Yellowstone (subband 71), Fig. 5 for Cuprite (subband 66), Fig. 6 for Indiana Pine (subband 4), Fig. 7 for Washington DC (subband 11), Fig. 8 for Mt. St. Helens (subband 19) and Fig. 9 for Erta Ale (subband 21) datasets. The original images can be compared to reconstructed images with the three SVR kernels. By visual comparisons of reconstructed images, it is observed that almost regions of entire images scenes are provided with good visual image quality. However, we also observed that many sub-bands of reconstructed images, with Polynomial and Linear kernels, present significant distortions (see Fig. 10) especially when the $\epsilon$ is over 0.0001. These results are consistent with those given 
by Table. 1. The reconstructed images with Gaussian kernel present less noisy and show more details than the other reconstructed images (see Fig. 10).

On Table. 1, experimental results show that our HSI compression algorithm achieves high compression ratios with a good image quality. Indeed, with the Yellowstone data set, we obtain a PSNR $=40.68 \mathrm{~dB}$ for $\mathrm{SSIM}=0.75$ and $\mathrm{CR}=26.44$ corresponding to $0.6 \mathrm{bpp}$. For Cuprite data, we obtain PSNR $=43.62 \mathrm{~dB}$ for $\mathrm{SSIM}=0.93$ and $\mathrm{CR}=26.95$ corresponding to $0.59 \mathrm{bpp}$, for Indiana Pines $\mathrm{PSNR}=44.14 \mathrm{~dB}$ for $\mathrm{SSIM}=0.85$. and $\mathrm{CR}=$ 27.02 corresponding to $0.59 \mathrm{bpp}$ and for Washington $\mathrm{DC}$ PSNR $=40.76 \mathrm{~dB}$ for $\mathrm{SSIM}=0.83$. and $\mathrm{CR}=26.95$ corresponding to $0.59 \mathrm{bpp}$. Results for Hyperion M.S. Helens dataset show a PSNR $=49.47 \mathrm{~dB}$ for $\mathrm{SSIM}=$ 0.89 and $\mathrm{CR}=22.12$ corresponding to $0.72 \mathrm{bpp}$. For Hyperion Erta Ale a PSNR $=38.46 \mathrm{~dB}$ for $\mathrm{SSIM}=$ 0.86 and $\mathrm{CR}=20.85$ corresponding to $0.76 \mathrm{bpp}$ are obtained.

By varying the hyper-parameters of the Gaussian and Polynomial kernels used by SVM, PSNR and SSIM increase when $\epsilon$-SVR decreases according to a law which can be approximately exponential (Table . 1). The Gaussian kernel converges to the best PSNR and SSIM faster than the two other kernels. Indeed, as shown in Table . 1, results for all datasets with Gaussian kernel achieve best values for $\epsilon$-SVR $=0.001$, when results given by Polynomial and Linear kernels still bellow their best values with about $1 \mathrm{~dB}$ and 0.05 of PSNR and SSIM respectively for all airborne datasets. For the two spaceborne datasets, the best PSNRs and SSIMs given by Polynomial and Linear kernels are lower than those given by Gaussian kernel with about $3 \mathrm{~dB}$.

In general, we can see that the developed approach, whether with Gaussian or Polynomial SVM kernels, leads to interesting result compression. In terms of quality, one can conclude that this approach preserves most significant part of information.

\subsection{Discussions on DWT filters}

Table. 2 shows results of our compression approach with four wavelet functions. The minimum PSNR are: 26.09 $\mathrm{dB}, 29.04 \mathrm{~dB}, 29.70 \mathrm{~dB}, 27.72 \mathrm{~dB}, 19.72 \mathrm{~dB}$ and 20.06 $\mathrm{dB}$ for Yellowstone, Cuprite, Indiana Pines, Washington DC, M.S.Helens and Erta Ale datasets respectively, with a bit rate 0.1 , and they belong to $\mathrm{Db} 4$ wavelet filter, while the maximum PSNR: $42.41 \mathrm{~dB}, 47.62 \mathrm{~dB}$, $44.14 \mathrm{~dB}, 44.76 \mathrm{~dB}, 49.47 \mathrm{~dB}$ and $38.46 \mathrm{~dB}$ belong to CDF $7 / 9$ wavelet filter. The CDF $7 / 9$ wavelet filter outperforms the other wavelet functions.

\subsection{Comparison results with others approaches}

We compared the SNR obtained with our compression process with those obtained by [42] for Indiana Pines and Washington DC images and [43] for Cuprite image. Fig. 11 shows the comparison results of our algorithm and the other approaches applied on Cuprite (256 x 256 x 128), Indiana Pines (145 x 145 x 200) and Washington DC ( $280 \times 307 \times 191)$ datasets. Note that dimensions of the taken images are similar to those taken by the cited references bellow. The compression performance is substantially improved by the proposed 3DDWT+SVR method, when compared to other methods such as: 3D-SPECK [42-44], PCA+JPEG2000 [42, 43, 45, 46], SSASR [42], JPEG-BIFR [42, 47], 3D-DCT $[43,48]$ and 3D-DCT+SVM [43] algorithms.

The comparison of our approach with the approaches 3D-DCT +SVM and SSASR demonstrates competitive results in term of performance and outperforms the other approaches. The developed approach achieves high compression ratios with good decoded image quality.

\subsection{Computational time}

The execution times of our method is evaluated in this section. The computational time of the used HSI data are shown In Table. 3. For all datasets the execution time increases with the bit rates. The taken times depend on the sizes and distributions of data.

Furthermore, results of the proposed approach, applied on Indiana Pines, are compared to those given by [42] ( Table. 4). The methods in [42] are executed with Intel Core CPU processors of $2.50 \mathrm{GHz}$ and 16GB RAM. PWSR and SSASR [42] methods are implemented on the MATLAB platform and 3D-SPECK [42-44], JPEG-BIFR [42, 47] and PCA+JPEG2000 [42, $43,45,46]$ methods are implemented by the $C / C++$ code.

The results show that the computational time obtained using our approach for various bit rates is very efficient for the lowest bit rates and increases considerably for the bit rates: 0.5 to 1 . This is due to the number of SVs that increases when SVR parameters are changed. However the execution time can be considerably improved with the use of a processor with better performances and a parallel computation.

\section{Classification performances}

To evaluate the effect of our method on classification performances, we use rbf-SVM classifier as in [42, 46, 
Table 1: Experimental results for Gaussian $(\mathrm{G})$, polynomial $(\mathrm{P})$ and linear (L) SVR kernels.

\begin{tabular}{|c|c|c|c|c|c|c|c|c|c|}
\hline Image & Kernels & $\epsilon-\mathrm{SVR}$ & 0.1 & 0.05 & 0.01 & 0.003 & 0.001 & 0.00001 & 1 e- 6 \\
\hline \multirow{9}{*}{ Yellowstone } & \multirow{3}{*}{$\mathrm{L}$} & PSNR (dB) & 23.32 & 31.18 & 31.18 & 33.98 & 29.73 & 39.90 & 40.68 \\
\hline & & Bit rate & 0.47 & 1.57 & 1.06 & 2.21 & 1.59 & 0.61 & 1.21 \\
\hline & & SSIM & 0.07 & 0.11 & 0.29 & 0.48 & 0.64 & 0.74 & 0.74 \\
\hline & \multirow{3}{*}{$\mathrm{P}$} & PSNR (dB) & 22.06 & 24.10 & 30.46 & 35.09 & 38.24 & 40.68 & 40.68 \\
\hline & & Bit rate & 0.38 & 0.52 & 0.59 & 0.60 & 0.60 & 0.60 & 0.60 \\
\hline & & SSIM & 0.05 & 0.09 & 0.25 & 0.48 & 0.67 & 0.75 & 0.75 \\
\hline & \multirow{3}{*}{ G } & PSNR (dB) & 26.01 & 29.49 & 31.65 & 40.65 & 40.68 & 40.68 & 40.68 \\
\hline & & Bit rate & 0.46 & 0.51 & 0.52 & 0.60 & 0.60 & 0.60 & 0.60 \\
\hline & & SSIM & 0.12 & 0.17 & 0.51 & 0.74 & 0.75 & 0.75 & 0.75 \\
\hline \multirow{9}{*}{ Cuprite } & \multirow{3}{*}{$\mathrm{L}$} & PSNR & 29.84 & 31.19 & 36.65 & 40.72 & 42.64 & 43.62 & 43.62 \\
\hline & & Bit rate & 0.40 & 0.48 & 0.57 & 0.59 & 0.59 & 0.59 & 0.59 \\
\hline & & SSIM & 0.07 & 0.10 & 0.35 & 0.66 & 0.83 & 0.92 & 0.92 \\
\hline & \multirow{3}{*}{$\mathrm{P}$} & PSNR & 29.18 & 30.62 & 36.84 & 41.00 & 42.92 & 43.62 & 43.62 \\
\hline & & Bit rate & 0.36 & 0.46 & 0.57 & 0.59 & 0.59 & 0.59 & 0.59 \\
\hline & & SSIM & 0,06 & 0,09 & 0,37 & 0,69 & 0,86 & 0,93 & 0.93 \\
\hline & \multirow{3}{*}{ G } & PSNR & 29.20 & 32.06 & 39.16 & 43.57 & 43.62 & 43.62 & 43.62 \\
\hline & & Bit rate & 0.33 & 0.49 & 0.58 & 0.59 & 0.59 & 0.59 & 0.59 \\
\hline & & SSIM & 0.08 & 0.15 & 0.51 & 0.93 & 0.93 & 0.93 & 0.93 \\
\hline \multirow{9}{*}{ Indiana } & \multirow{3}{*}{$\mathrm{L}$} & PSNR & 21.51 & 23.78 & 29.26 & 31.32 & 31.96 & 32.14 & 32.14 \\
\hline & & Bit rate & 0.42 & 0.51 & 0.58 & 0.59 & 0.59 & 0.59 & 0.59 \\
\hline & & SSIM & 0.11 & 0.17 & 0.47 & 0.70 & 0.81 & 0.84 & 0.84 \\
\hline & \multirow{3}{*}{$\mathrm{P}$} & PSNR & 32.87 & 35.26 & 41.18 & 43.36 & 44.04 & 44.14 & 44.14 \\
\hline & & Bit rate & 0.39 & 0.50 & 0.41 & 0.60 & 0.59 & 0.59 & 0.59 \\
\hline & & SSIM & 0.09 & 0.16 & 0.48 & 0.72 & 0.83 & 0.85 & 0.85 \\
\hline & \multirow{3}{*}{ G } & PSNR & 32.70 & 35.97 & 42.39 & 44.12 & 44.14 & 44.14 & 44.14 \\
\hline & & Bit rate & 0.36 & 0.50 & 0.58 & 0.59 & 0.59 & 0.59 & 0.59 \\
\hline & & SSIM & 0.12 & 0.21 & 0.59 & 0.85 & 0.85 & 0.85 & 0.85 \\
\hline \multirow{9}{*}{ Washington } & \multirow{3}{*}{$\mathrm{L}$} & PSNR & 26.88 & 28.50 & 33.84 & 37.97 & 39.79 & 40.76 & 40.76 \\
\hline & & Bit rate & 0.40 & 0.50 & 0.58 & 0.59 & 0.59 & 0.59 & 0.59 \\
\hline & & SSIM & 0.07 & 0.11 & 0.33 & 0.58 & 0.73 & 0.82 & 0.82 \\
\hline & \multirow{3}{*}{$\mathrm{P}$} & PSNR & 26.29 & 27.93 & 34.00 & 38.12 & 40.15 & 40.76 & 40.76 \\
\hline & & Bit rate & 0.36 & 0.48 & 0.58 & 0.59 & 0.59 & 0.59 & 0.59 \\
\hline & & SSIM & 0.05 & 0.09 & 0.33 & 0.60 & 0.77 & 0.83 & 0.83 \\
\hline & \multirow{3}{*}{$\mathrm{G}$} & PSNR & 25.83 & 28.66 & 36.11 & 40.72 & 40.76 & 40.76 & 40.76 \\
\hline & & Bit rate & 0.34 & 0.50 & 0.58 & 0.59 & 0.59 & 0.59 & 0.59 \\
\hline & & SSIM & 0.05 & 0.11 & 0.44 & 0.82 & 0.83 & 0.83 & 0.83 \\
\hline \multirow{9}{*}{ M.S. Helens } & & PSNR & 21.38 & 21.60 & 21.68 & 22.06 & 33.62 & 33.75 & 45.61 \\
\hline & $\mathrm{L}$ & Bit rate & 0.45 & 0.56 & 0.58 & 0.61 & 0.61 & 0.69 & 0.72 \\
\hline & & SSIM & 0.18 & 0.25 & 0.26 & 0.34 & 0.37 & 0.60 & 0.85 \\
\hline & & PSNR & 22.79 & 22.79 & 22.79 & 22.99 & 34.61 & 34.70 & 46.62 \\
\hline & $\mathrm{P}$ & Bit rate & 0.60 & 0.60 & 0.60 & 0.61 & 0.61 & 0.69 & 0.72 \\
\hline & & SSIM & 0.10 & 0.10 & 0.10 & 0.36 & 0.82 & 0.83 & 0.86 \\
\hline & & PSNR & 24.42 & 24.50 & 24.58 & 47.25 & 49.47 & 49.47 & 49.47 \\
\hline & G & Bit rate & 0.48 & 0.59 & 0.60 & 0.69 & 0.72 & 0.72 & 0.72 \\
\hline & & SSIM & 0.26 & 0.29 & 0.31 & 0.80 & 0.89 & 0.89 & 0.89 \\
\hline & & PSNR & 20.18 & 20.19 & 20.19 & 22.70 & 22.71 & 23.52 & 32.51 \\
\hline & $\mathrm{L}$ & Bit rate & 0.45 & 0.59 & 0.59 & 0.66 & 0.71 & 0.71 & 0.72 \\
\hline & & SSIM & 0.21 & 0.21 & 0.43 & 0.43 & 0.68 & 0.71 & 0.82 \\
\hline & & PSNR & 21.91 & 21.91 & 21.93 & 22.71 & 22.73 & 24.87 & 36.58 \\
\hline Erta Ale & $\mathrm{P}$ & Bit rate & 0.42 & 0.50 & 0.59 & 0.73 & 0.75 & 0.75 & 0.76 \\
\hline & & SSIM & 0.22 & 0.22 & 0.43 & 0.55 & 0.69 & 0.76 & 0.82 \\
\hline & & PSNR & 22.47 & 22.50 & 22.76 & 24.74 & 38.48 & 38.48 & 38.46 \\
\hline & G & Bit rate & 0.43 & 0.59 & 0.62 & 0.66 & 0.76 & 0.76 & 0.76 \\
\hline & & SSIM & 0.23 & 0.23 & 0.54 & 0.68 & 0.86 & 0.86 & 0.86 \\
\hline
\end{tabular}




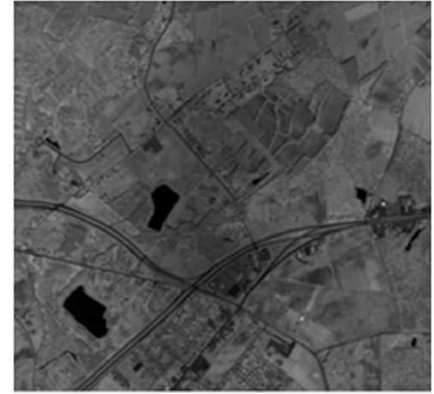

(a)

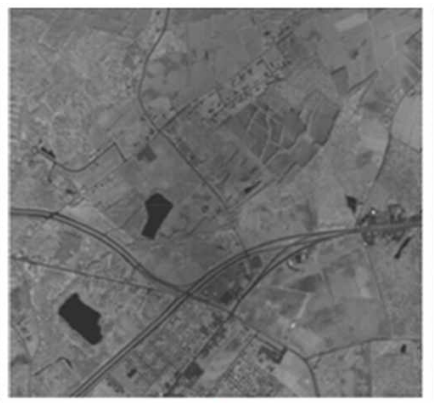

(b)

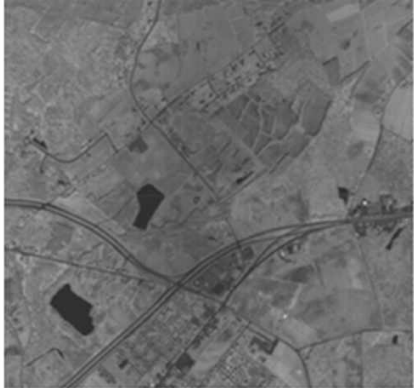

(c)

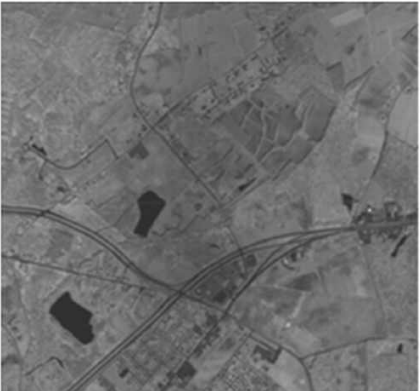

(d)

Fig. 4: Compression results for Yellowstone:

(a) Yellowstone original image, reconstructed images with CR close to 26 with (b)Gaussian, (c)polynomial and (d)linear kernels.

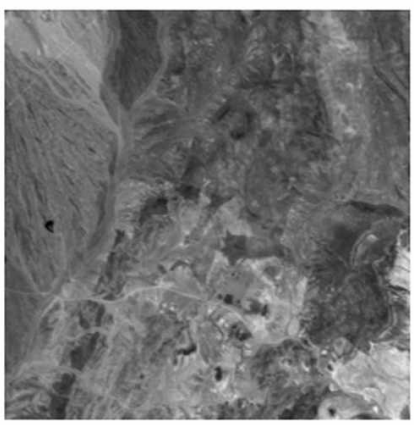

(a)

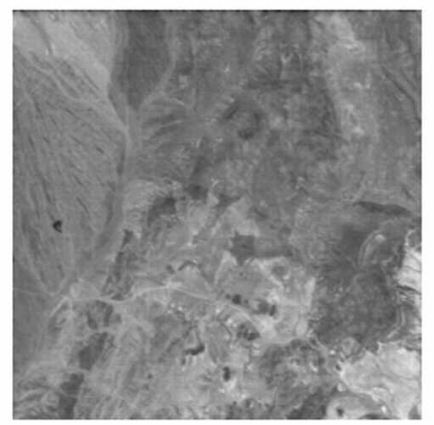

(b)

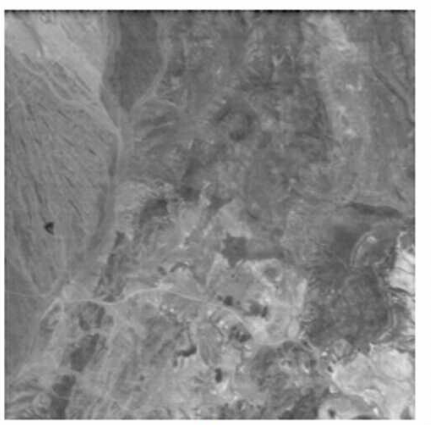

(c)

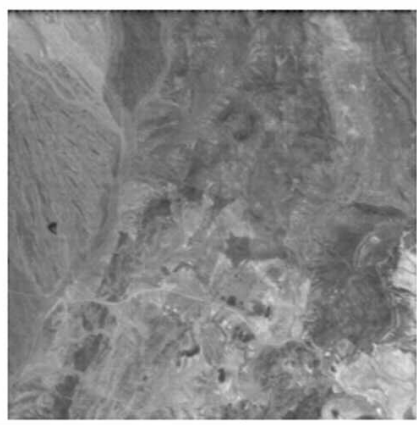

(d)

Fig. 5: Compression results for Cuprite scene:

(a) Cuprite original image, reconstructed images with CR close to 27 with (b)Gaussian, (c)polynomial and (d)linear kernels.

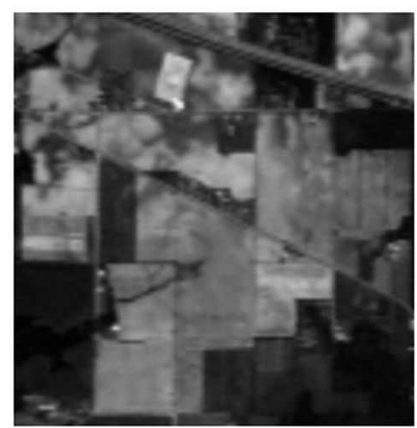

(a)

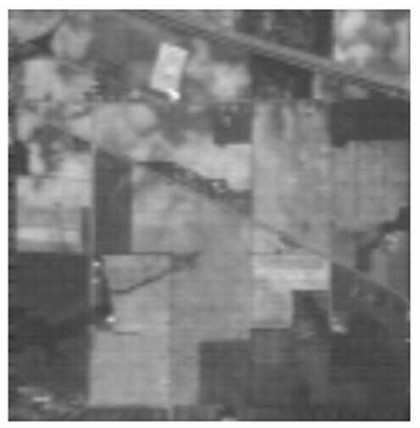

(b)

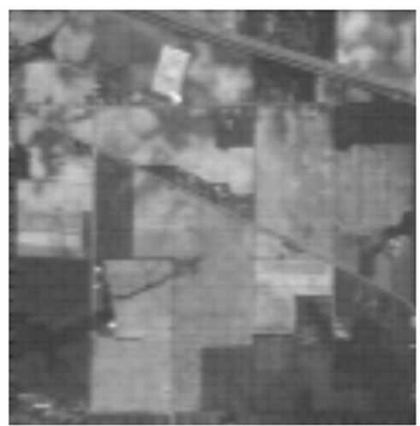

(c)

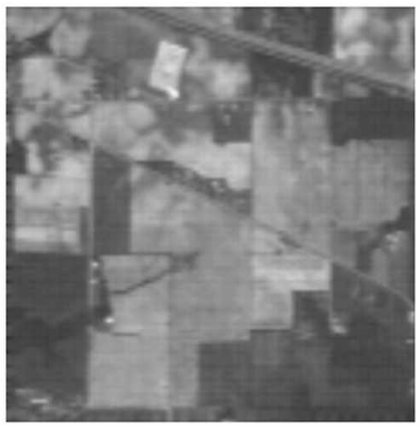

(d)

Fig. 6: Compression results for Indiana Pines scene:

(a) Indiana Pines original image, reconstructed images with CR close to 39 with (b)Gaussian, (c)polynomial and (d)linear kernels.

47, 49] . We apply the classifier on originals and reconstructed images over the four airborne HSI datasets, which contain rich variety of information. The ground truth images of Indiana Pines (with 16 classes and 10366 samples) and Washington DC ( with 7 classes and 7869 samples) are available online ${ }^{7}$. For Yellowstone and Cuprite scenes, we used ENVI to create the labeled pixels. The classes number of Cuprite ground truth image

\footnotetext{
7 http:www.ehu.eus/ccwintco/index.php?title= Hyperspectral_Remote_Sensing_Scenes, http://lesun. weebly.com/hyperspectral-data-set.html
} 


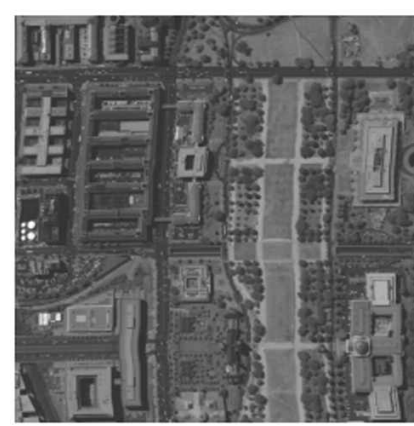

(a)

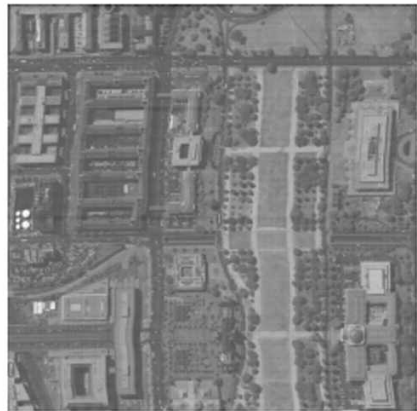

(b)

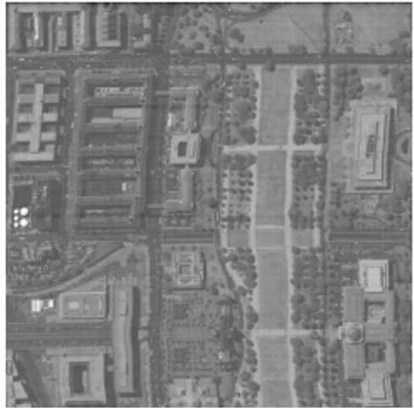

(c)

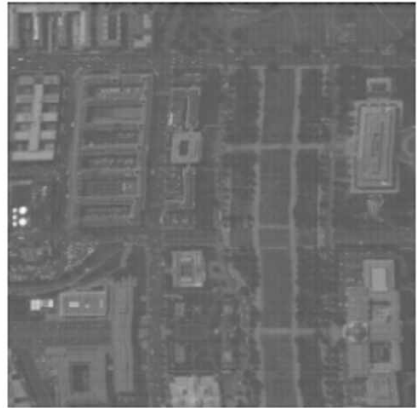

(d)

Fig. 7: Compression results for Washington DC scene :

(a) Washington DC original image, reconstructed images with CR close to 27 with (b)Gaussian, (c)polynomial and (d)linear kernels.

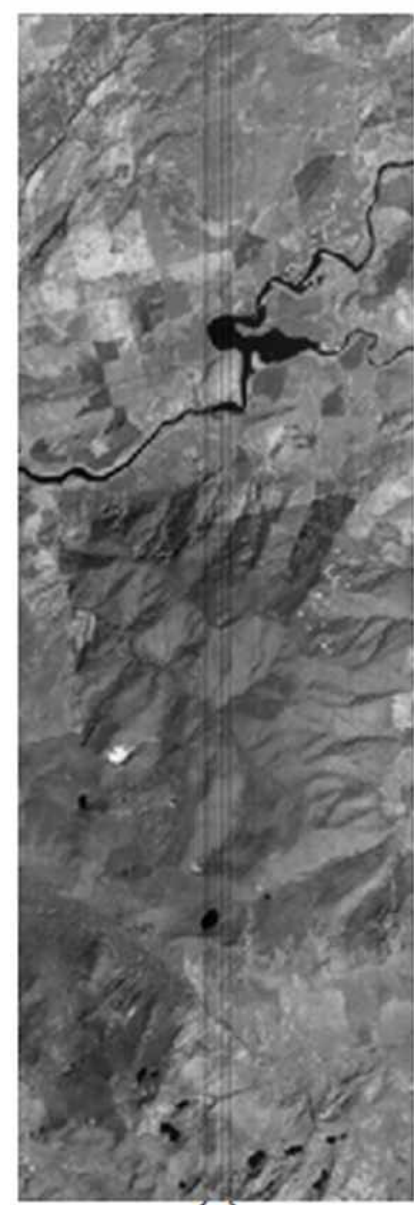

(a)

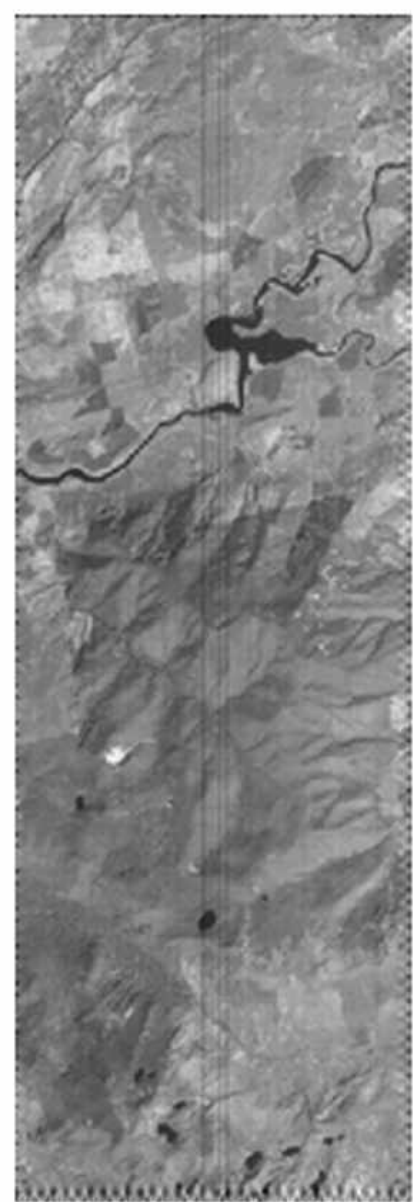

(b)

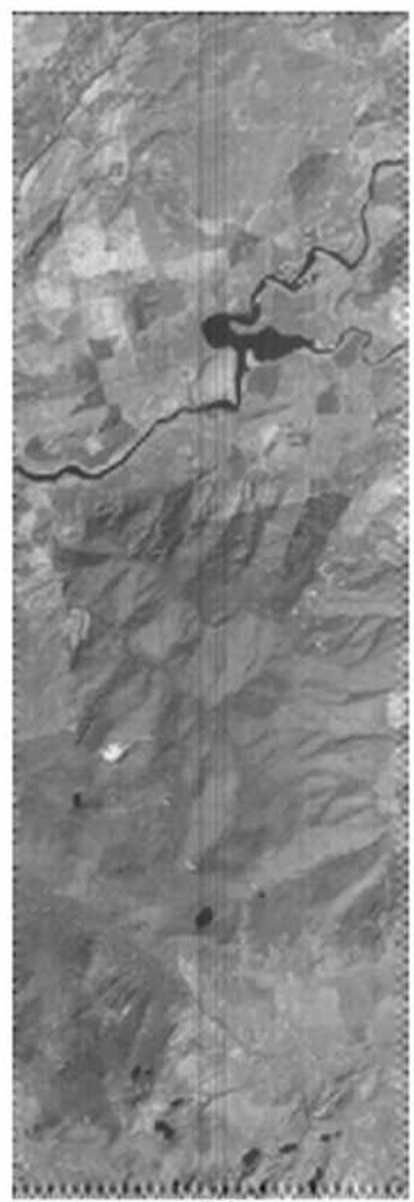

(c)

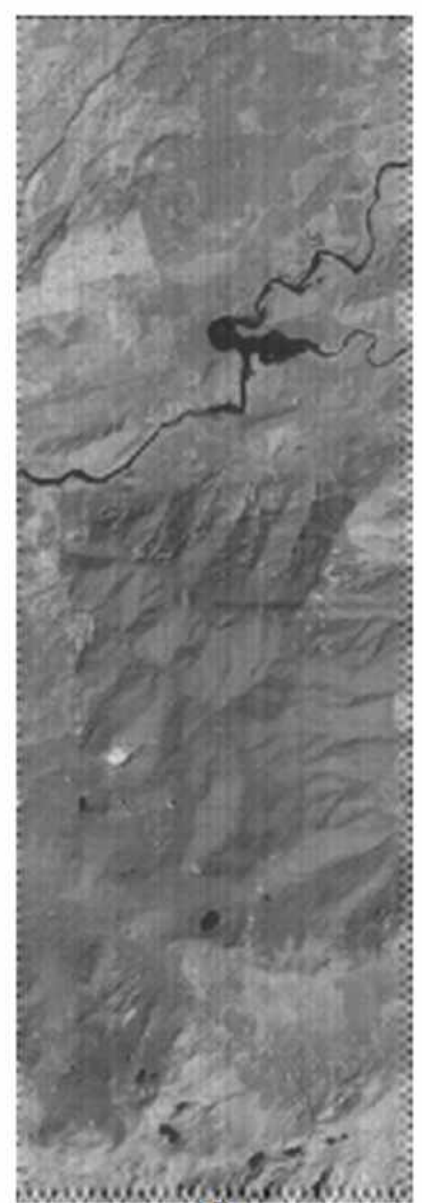

(d)

Fig. 8: Compression results for M.S.Helens image :

(a) M.S.Helens original image, reconstructed images with CR close to 23 with (b)Gaussian (PSNR=49.47 dB), (c) polynomial $(\mathrm{PSNR}=46.62 \mathrm{~dB})$ and $(\mathrm{d})$ linear $((\mathrm{PSNR}=45.61 \mathrm{~dB})$ kernels.

is fixed to 12 by reducing the number of minerals witch represent similar proprieties (initially Cuprite image represents 14 classes) ${ }^{8}$. For Yellowstone ground refer-

\footnotetext{
8 http://www.escience.cn/people/feiyunZHU/Dataset_ GT .html
} 


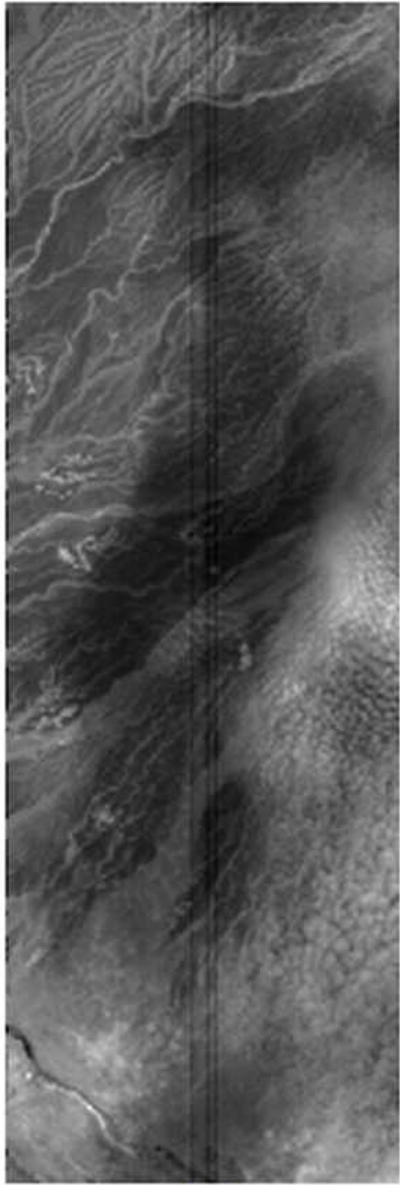

(a)

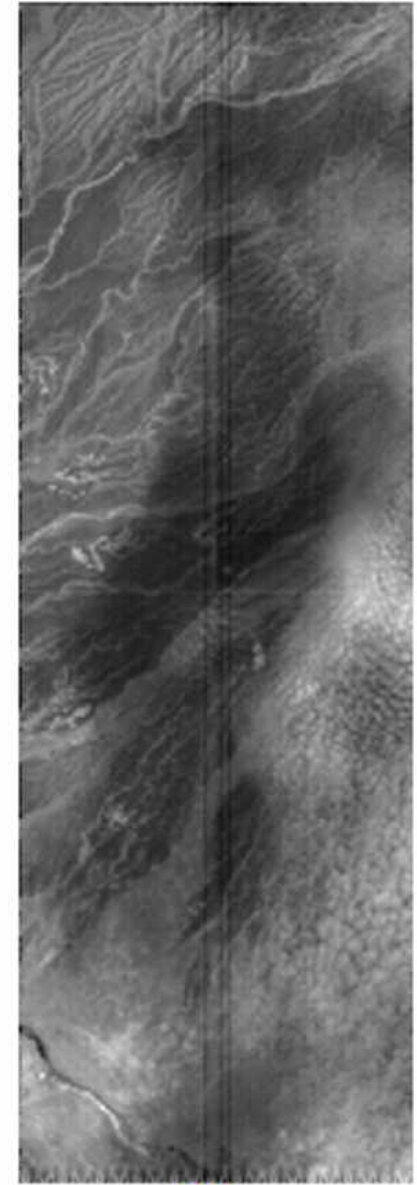

(b)

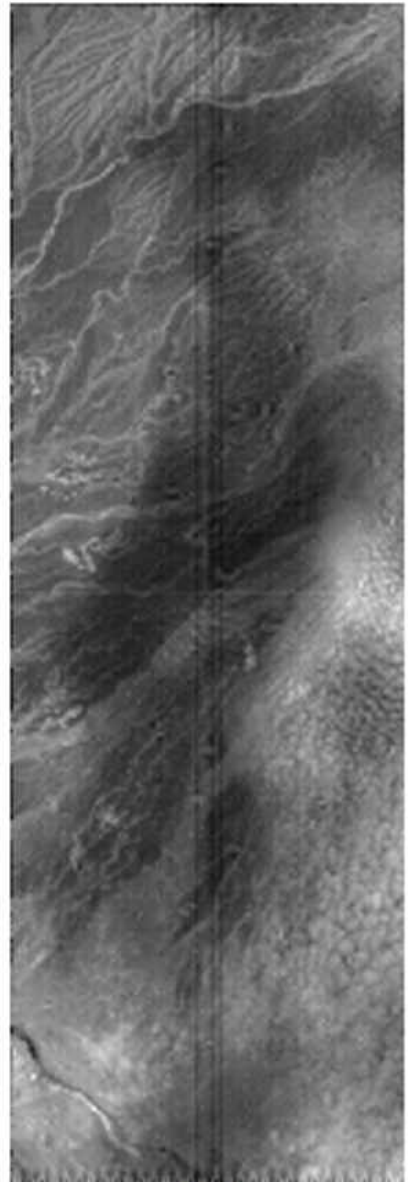

(c)

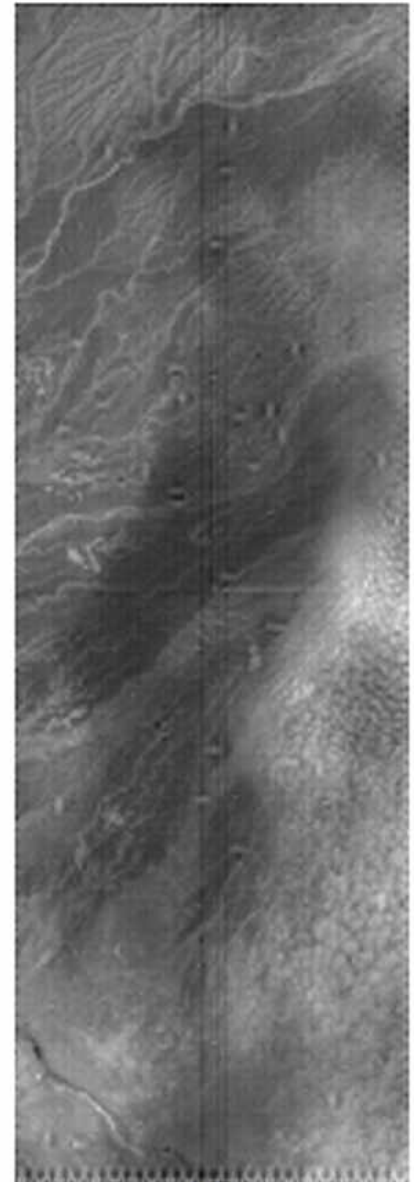

(d)

Fig. 9: Compression results for Erta Ale image :

(a) Erta Ale original image, reconstructed images with CR close to 22 with (b)Gaussian (PSNR=38.46 dB), (c) Polynomial $(\mathrm{PSNR}=36.58 \mathrm{~dB})$ and $(\mathrm{d})$ Linear $((\mathrm{PSNR}=32.51 \mathrm{~dB})$ kernels.

ence, we have considered 5 classes. The training samples number of HSI datasets is fixed to about $5 \%$ for each class. The rest of samples represents the test samples. In table 5, we give Overall Accuracy (OA), Average Accuracy (AA) and Kappa coefficient $(K)$ for Bit rates of $0.1,0.2,0.3,0.4,0.5,0.6,0.7,0.8,0.9,1.0$ (bppb) of the six HSI datasets. In the same table, classification performances results with SVM classifier applied on original HSI images and 2D-DWT + SVR compressed data are provided. This last compression method consists in performing a 2D-DWT and SVR over all bands (band per band) of HSI image [50]. The best classification results obtained with 2D-DWT + SVR method appear for bit rates of $0.5 \mathrm{bppb}$ for Yellowstone, $0.1 \mathrm{bppb}$ for Cuprite and $1 \mathrm{bppb}$ for Indiana and Washington datasets. Classification results are shown in table 5 .

We compare results given by the proposed method on Indiana Pines with those in [46]. The comparison is shown in Fig. 12. Results in table 5 show that the best classification performances appear for bit rate 0.4 bppb for Cuprite and Indiana Pines datasets and bit rates 0.2 and $0.5 \mathrm{bppb}$ for Yellowstone and Washington DC datasets respectively. In Fig. 12, one can see that except for bit rates of $0.1,0.2$ and $0.3 \mathrm{bppb}$, for which PCA+JPEG2000 and AR+PCA+JPEG2000 greatly outperform the other approaches. The proposed 3D-DWT + SVR method overcomes all compared methods, especially for bit rates from 0.4 to $1 \mathrm{bppb}$. Results given by 3D-DWT + SVR show the effectiveness of the method for classification purpose. In addition, one can observe that the classification performances for compressed images exceed those of original images. This phenomena is observed for all tested datasets. One can also observe that the proposed method overcomes the 2D-DWT + SVR in term of classification accuracy. To confirm the accuracy of the proposed approach for classification tasks, we also apply the McNemar's test on the method. Table 6 gives results of McNemar's test of 3D-DWT+SVR 


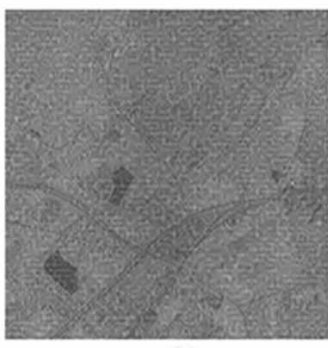

(a)

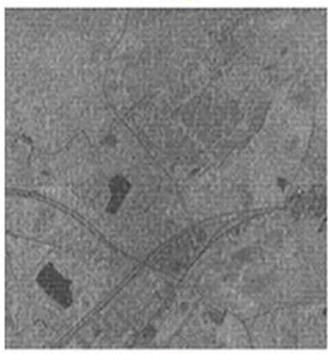

(d)

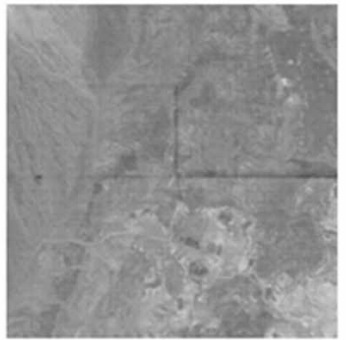

(b)

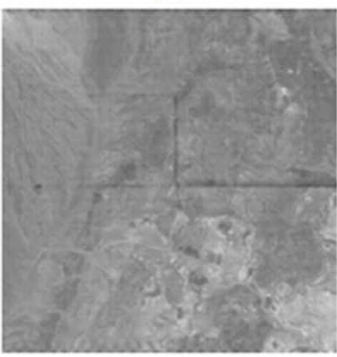

(e)

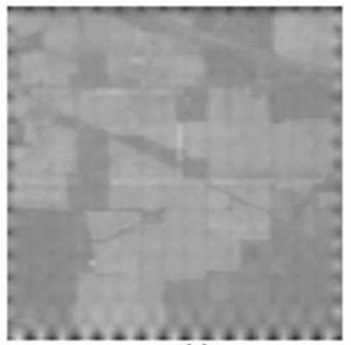

(c)

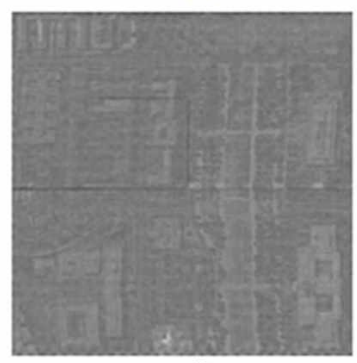

(f)

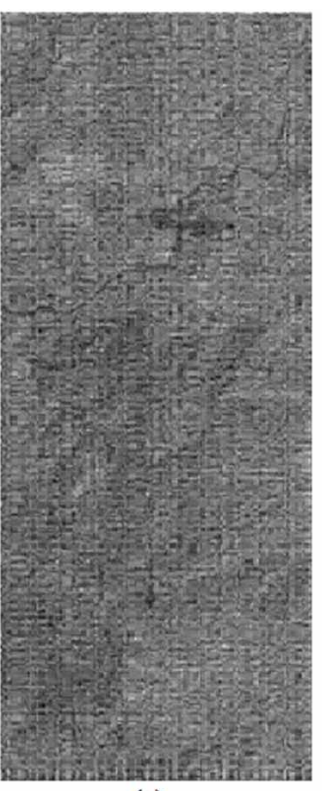

(g)

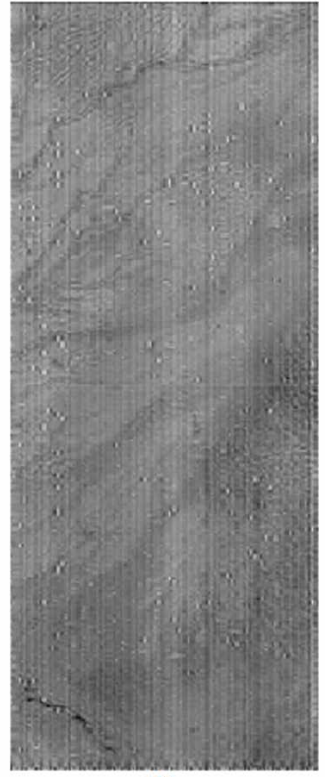

(h)

Fig. 10: Reconstructed images with linear and polynomial kernels:

Linear: (a)Yellowstone (subband 91), (b) Cuprite (subband 6), (c) Indiana Pines (subband 183 (g) M.S.Helens (subband 40)

Polynomial: (d) Yellowstone (subband 91), (e) Cuprite (subband 6), (f) Washington DC (subband 160) (h) Erta Ale (subband 93).
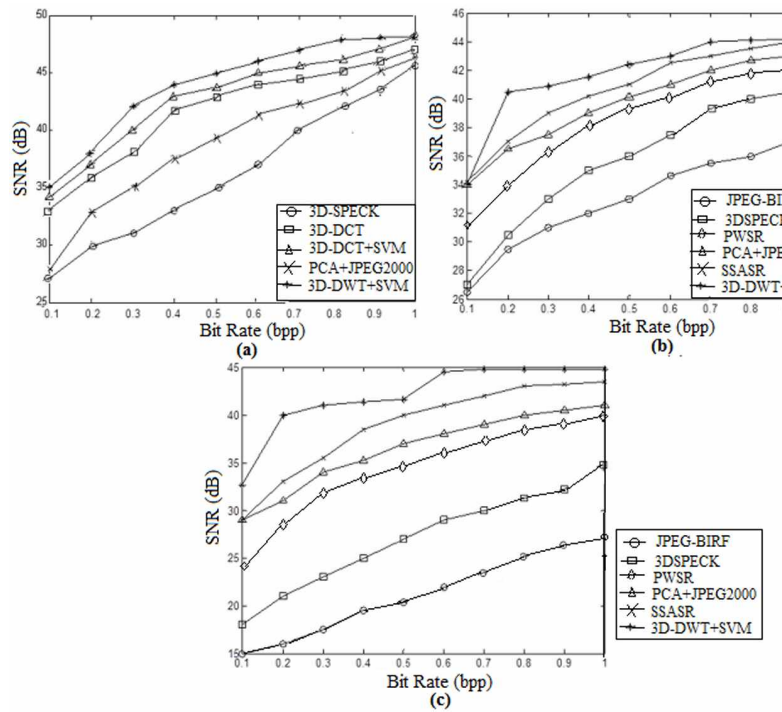

(c)

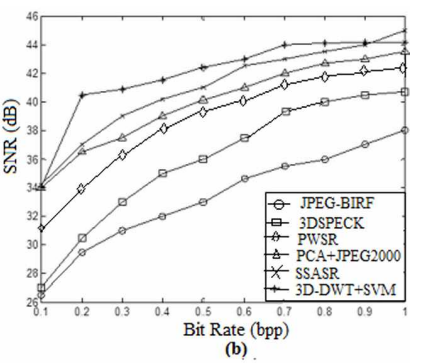

列

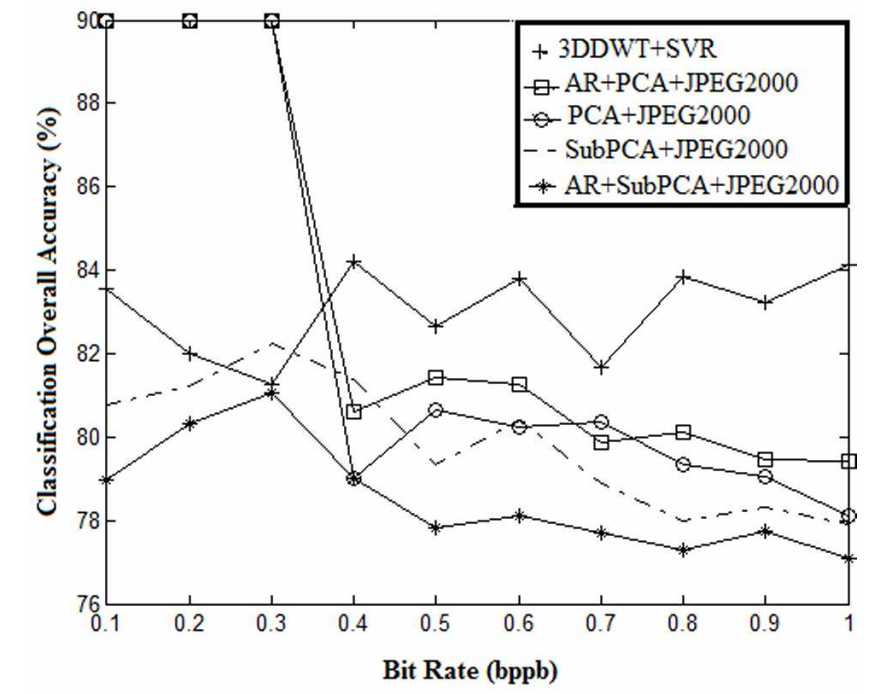

Fig. 12: Comparison of OA\% with famous compression methods over Indiana Pines. 
Table 2: Compression results of the proposed method with different wavelet filters

\begin{tabular}{|c|c|c|c|c|c|c|}
\hline \multirow{2}{*}{ Image } & \multicolumn{6}{|c|}{ PSNR (dB) for the proposed approach } \\
\hline & Wavelet I & action & CDF $9 / 7$ & $\mathrm{CDF} 5 / 3$ & $\mathrm{Db} 4$ & Sym \\
\hline \multirow{5}{*}{ Yellowstone } & \multirow{5}{*}{ Bit rate } & 0.1 & 28.20 & 27.49 & 26.09 & 26.78 \\
\hline & & 0.25 & 31.81 & 30.25 & 29.85 & 30.09 \\
\hline & & 0.5 & 39.79 & 39.64 & 38.57 & 38.87 \\
\hline & & 0.75 & 42.34 & 41.75 & 41.29 & 41.35 \\
\hline & & 1 & 42.41 & 41.81 & 41.41 & 41.60 \\
\hline \multirow{5}{*}{ Cuprite } & \multirow{5}{*}{ Bit rate } & 0.1 & 35.20 & 30.80 & 29.04 & 29.78 \\
\hline & & 0.25 & 40.06 & 39.35 & 38.94 & 39.11 \\
\hline & & 0.50 & 45.16 & 40.09 & 37.88 & 39.71 \\
\hline & & 0.75 & 46.78 & 44.06 & 41.95 & 42.48 \\
\hline & & 1 & 47.62 & 44.13 & 42.03 & 42.53 \\
\hline \multirow{5}{*}{ Indiana Pines } & \multirow{5}{*}{ Bit rate } & 0.1 & 34.02 & 30.55 & 29.70 & 30.52 \\
\hline & & 0.25 & 40.7 & 39.14 & 38.82 & 39.02 \\
\hline & & 0.50 & 42.39 & 42.30 & 41.76 & 42.10 \\
\hline & & 0.75 & 44.11 & 43.52 & 43.39 & 43.41 \\
\hline & & 1 & 44.14 & 43.60 & 43.42 & 43.5 \\
\hline \multirow{5}{*}{ Washington DC } & \multirow{5}{*}{ Bit rate } & 0.1 & 32.66 & 30.80 & 27.72 & 28.44 \\
\hline & & 0.25 & 40.11 & 39.35 & 31.09 & 31.51 \\
\hline & & 0.50 & 41.66 & 40.09 & 37.39 & 39.47 \\
\hline & & 0.75 & 44.72 & 44.06 & 41.93 & 42.22 \\
\hline & & 1 & 44.76 & 44.13 & 42.09 & 42.53 \\
\hline \multirow{5}{*}{ M.S. Helens } & \multirow{5}{*}{ Bit rate } & 0.1 & 22.42 & 22.06 & 19.72 & 19.92 \\
\hline & & 0.25 & 24.22 & 23.71 & 21.33 & 22.76 \\
\hline & & 0.50 & 24.50 & 23.80 & 21.58 & 22.76 \\
\hline & & 0.75 & 49.47 & 34.06 & 24.35 & 33.52 \\
\hline & & 1 & 49.47 & 42.23 & 36.62 & 41.79 \\
\hline \multirow{5}{*}{ Erta Ale } & \multirow{5}{*}{ Bit rate } & 0.1 & 20.72 & 20.47 & 20.06 & 20.20 \\
\hline & & 0.25 & 22.40 & 21.70 & 20.06 & 20.26 \\
\hline & & 0.50 & 22.50 & 21.92 & 20.52 & 21.64 \\
\hline & & 0.75 & 38.46 & 36.66 & 25.32 & 23.59 \\
\hline & & 1 & 38.46 & 36.69 & 25.36 & 24.15 \\
\hline
\end{tabular}

Table 3: Execution time (s) of 3D-DWT + SVR

\begin{tabular}{|c|c|c|c|c|c|c|}
\hline Rate (bpp) & Yellowstone & Cuprite & Indiana & Washington & M.S.Helens & Erta Ale \\
\hline 0.1 & 122.82 & 54.06 & 7.02 & 49.15 & 284.70 & 227.75 \\
0.2 & 178.52 & 190.11 & 8.50 & 154.06 & 303.64 & 258.60 \\
0.3 & 191.37 & 132.63 & 26.28 & 190.16 & 357.17 & 269.84 \\
0.4 & 324.28 & 262.42 & 52.29 & 235.16 & 417.22 & 294.61 \\
0.5 & 402.81 & 318.40 & 65.20 & 325.98 & 771.30 & 416.80 \\
0.6 & 917.50 & 772.94 & 160.34 & 540.67 & 820.42 & 522.77 \\
0.7 & 1376.25 & 998.41 & 870.95 & 770.04 & 1253.36 & 1049.90 \\
0.8 & 1634.76 & 1293.23 & 895.05 & 802.81 & 1313.34 & 1078.81 \\
0.9 & 1658.71 & 1606.71 & 967.15 & 950.27 & 2247.12 & 2084.01 \\
1 & 1985.88 & 1645.34 & 1032.01 & 1608.60 & 2747.97 & 2613.50 \\
\hline
\end{tabular}

fication such as [51-54]. These classifiers demonstrated their effectiveness in HSI classification field and out- performed many known effective classifiers. Comparison classification accuracy of the proposed method with 
Table 4: Comparison of execution time (s) over Indiana Pines

\begin{tabular}{|c|c|c|c|c|c|c|c|c|c|c|c|}
\hline \hline \multirow{3}{*}{ Code } & & \multicolumn{10}{|c|}{ Bit Rate } \\
\cline { 3 - 12 }$C / C++$ & Method & 0.1 & 0.2 & 0.3 & 0.4 & 0.5 & 0.6 & 0.7 & 0.8 & 0.9 & 1.0 \\
\hline \multirow{3}{*}{ MATLAB } & 3D SPECK & 20.3 & 2.24 & 2.58 & 2.84 & 3.21 & 2.52 & 2.72 & 4.07 & 4.35 & 4.67 \\
& JPEG-BIFR & 1.88 & 1.74 & 1.68 & 2.08 & 1.97 & 2.06 & 1.93 & 2.04 & 1.98 & 1.91 \\
& JPEG-2000 & 2.28 & 2.06 & 2.12 & 2.20 & 2.43 & 2.54 & 2.87 & 2.90 & 2.82 & 3.06 \\
\hline & PWSR & 6.19 & 8.61 & 12.2 & 16.9 & 21.3 & 28.5 & 36.1 & 43.0 & 50.8 & 60.7 \\
& SSASR & 7.13 & 7.61 & 8.57 & 9.35 & 10.1 & 11.0 & 12.3 & 13.9 & 15.3 & 17.1 \\
& 3D-DWT+ SVR & 7.02 & 8.50 & 26.28 & 52.29 & 65.20 & 160.34 & 870.96 & 895.06 & 967.15 & 1032.01 \\
\hline
\end{tabular}

Table 5: Classification performances of 3D-DWT + SVR applied over Yellowstone, Cuprite, Indiana Pines and Washington DC datasets

\begin{tabular}{|c|c|c|c|c|c|c|c|c|c|c|c|c|c|}
\hline \multirow{3}{*}{ Dataset } & \multirow{3}{*}{ Measure } & \multirow{3}{*}{$\begin{array}{l}\text { Original } \\
\text { Image }\end{array}$} & \multirow{3}{*}{$\begin{array}{c}2 \mathrm{D} \\
\mathrm{DWT} \\
+\mathrm{SVR} \\
\end{array}$} & \multicolumn{10}{|c|}{ 3D-DWT + SVR } \\
\hline & & & & \multicolumn{10}{|c|}{ Bit Rate } \\
\hline & & & & 0.1 & 0.2 & 0.3 & 0.4 & 0.5 & 0.6 & 0.7 & 0.8 & 0.9 & 1.0 \\
\hline \multirow{3}{*}{ Yellowstone } & OA \% & 91.20 & 91.35 & 91.02 & 91.55 & 90.86 & 91.31 & 90.37 & 89.78 & 91.17 & 89.40 & 88.84 & 89.28 \\
\hline & AA $\%$ & 87.58 & 88.85 & 87.75 & 88.97 & 87.18 & 88.02 & 85.97 & 85.20 & 87.34 & 84.36 & 83.17 & 84.11 \\
\hline & Карра & 0.84 & 0.85 & 0.84 & 0.85 & 0.83 & 0.84 & 0.82 & 0.81 & 0.84 & 0.80 & 0.79 & 0.80 \\
\hline \multirow{3}{*}{ Cuprite } & OA \% & 78.43 & 77.77 & 75.54 & 75.63 & 75.01 & 78.59 & 72.72 & 78.32 & 77.93 & 77.38 & 78.07 & 77.23 \\
\hline & $\mathrm{AA} \%$ & 77.26 & 76.45 & 74.12 & 74.17 & 73.15 & 77.28 & 70.43 & 77.02 & 76.67 & 75.94 & 76.83 & 75.74 \\
\hline & Kарра & 0.73 & 0.72 & 0.69 & 0.69 & 0.68 & 0.73 & 0.68 & 0.72 & 0.72 & 0.71 & 0.72 & 0.71 \\
\hline \multirow{3}{*}{ Indiana } & 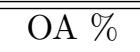 & 82.45 & 83.67 & 83.57 & 81.99 & 81.28 & 84.20 & 82.65 & 83.81 & 81.66 & 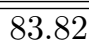 & 83.21 & $\overline{84.14}$ \\
\hline & AA $\%$ & 76.21 & 77.43 & 78.69 & 78.81 & 76.89 & 80.76 & 77.51 & 77.21 & 77.35 & 80.31 & 75.66 & 78.48 \\
\hline & Kappa & 0.79 & 0.81 & 0.80 & 0.79 & 0.78 & 0.81 & 0.79 & 0.80 & 0.79 & 0.81 & 0.80 & 0.81 \\
\hline \multirow{3}{*}{ Washington } & OA \% & 79.72 & 80.08 & 72.41 & 76.23 & 75.15 & 76.10 & 80.34 & 78.71 & $\overline{c 77.61}$ & 787.48 & 78.07 & 777.27 \\
\hline & AA $\%$ & 74.57 & 75.73 & 57.33 & 66.60 & 68.68 & 70.14 & 75.87 & 72.21 & 57.02 & 71.76 & 70.66 & 66.15 \\
\hline & Kappa & 0.78 & 0.79 & 0.65 & 0.69 & 0.73 & 0.74 & 0.79 & 0.77 & 0.76 & 0.76 & 0.76 & 0.75 \\
\hline
\end{tabular}

Table 6: McNemar's test (Z) of 3D-DWT + SVR over SVM and 2D-DWT + SVM methods.

\begin{tabular}{|c|c|c|}
\hline Dataset & $\begin{array}{c}\text { 3DDWT-SVR } \\
\text { vs } \\
\text { SVM }\end{array}$ & $\begin{array}{c}\text { 3DDWT-SVR } \\
\text { 2s } \\
\text { 2DDWT-SVM }\end{array}$ \\
\hline \hline Yellowstone & 6.70 & 2.23 \\
\hline Cuprite & 13.03 & 3.87 \\
\hline Indiana & 2.82 & 3.74 \\
\hline Washington & 16.76 & 3.87 \\
\hline
\end{tabular}

those of CNN-based classifiers given by [55] applied over Indiana Pines dataset with 200 training, yields to classification accuracies of $87.01 \%$ for CNN [51], $93.9 \%$ for CNN-PPF [53], 94.24\% for CD-CNN [56] and $98.54 \%$ for DR-CNN [55]. This presents differences of $2.81 \%$, $9.7 \%, 10.04 \%$ and $14.34 \%$ against our method.

\section{Impact on compressed reflectances}

We compress the Aviris Yellowstone and Washington DC images with spectra between 460nm and 2130nm and a compression ratio equal to 42.12 and 42.60 respectively. The regression is computed with a Gaussian kernel. We measured for 3D-DWT+SVR compressed image, PSNR(dB) of 30.21 (ground), 39.32 (rail), 31.57 (building) for Yellowstone scene and 27.13 (feild), 47.37 (building), 33.62 (ground) for Washington DC. In order to compare results with those of $2 \mathrm{D}-\mathrm{DWT}+\mathrm{SVR}$, PSNR (dB) of 30.78 (ground), 34.31(rail), 36.26 (building) for Yellowstone scene and 30.62 (field), 45.25 (building), 37.44 (ground) for Washington DC are also measured for 2D-DWT + SVR method . Figure. 13 shows the spectrum variation over bands for arbitrarily selected pixel blocks: ground, rail and building of Yellowstone image and field, building and ground of Washington DC image. For all the datasets, variations in the spectrum of the 3D-DWT + SVR compressed image are almost identical to those of the original image. Although there could be some differences between original 

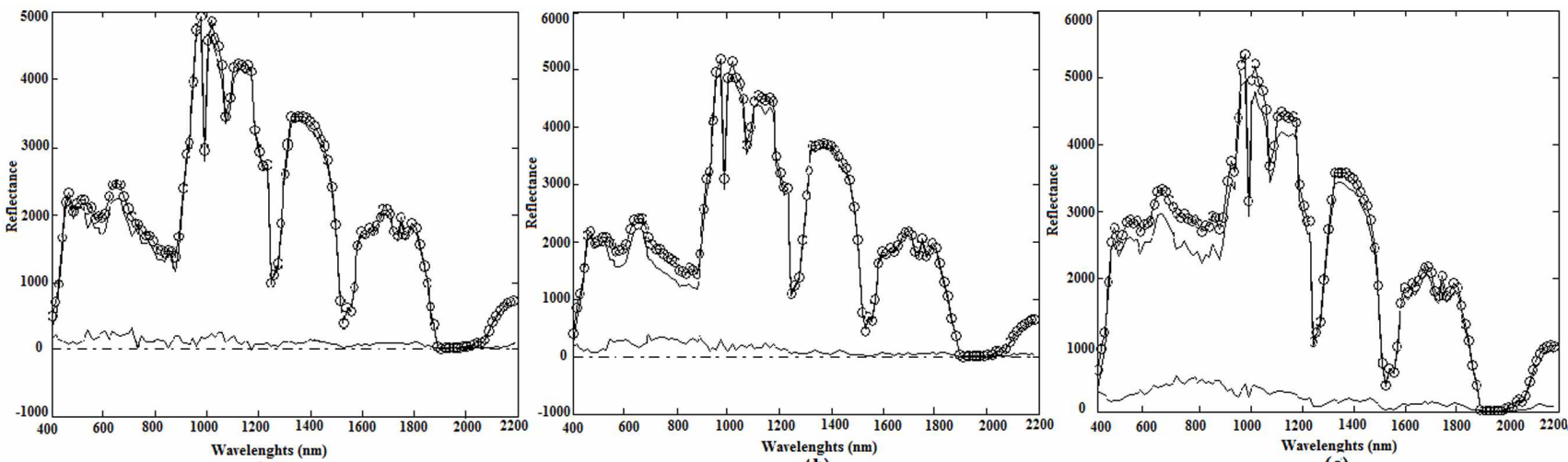

(a)

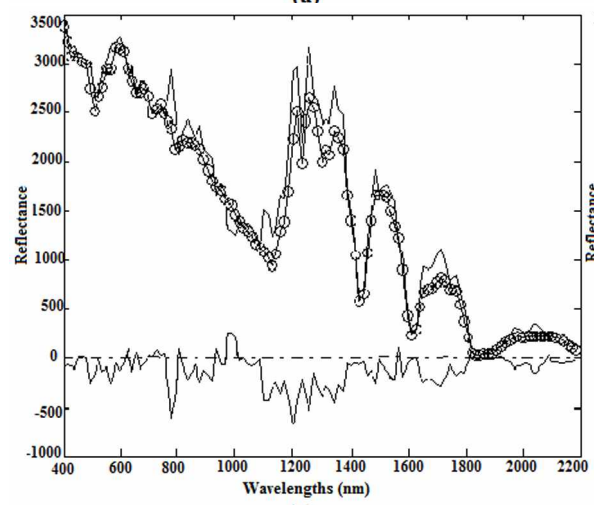

(b)

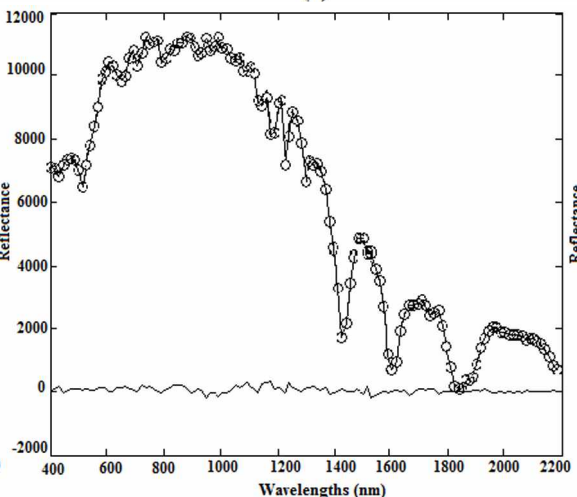

(e)

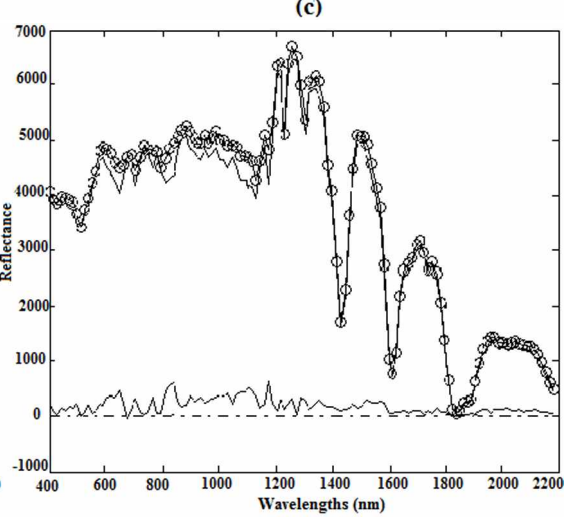

(f)

Fig. 13: Original and 3D-DWT + SVR compressed reflectances with their difference (symbole lines) and 2DDWT + SVR compressed reflectances with original reflectance difference (solid line) for a Gauss kernel: Yellowstone $(\mathrm{CR}=42.12)$, (a) ground , (b) rail , (c) building; Washington DC ( $\mathrm{CR}=42.60)$, (d) field, (e) building, (f) ground.

and compressed data, it is reasonable to conclude that the spectral information is preserved using our compression method. Despite a high compression ratio, the PSNR between compressed spectra and corresponding original spectra are correct. Thus, results support those presented obviously witch demonstrate the effectiveness of the method for classification-oriented applications. This may allow good separation of classes and the presentation of the almost part of information present in the image. Furthermore, a comparison of spectrum variation with 2D-DWT + SVR method over the selected blocks and the difference between results given by 3DDWT + SVR and 2D-DWT + SVR are shown in the Figure. 13. Results, show that the proposed method presents more spectral fidelity than the 2D-DWT + SVR.

\section{Conclusion}

An effective hyperspectral compression method, based on 3D wavelets transform and spectrum learning with regression vector machine, is proposed in the current work to efficiently exploit the spectral and the spatial redundancies in HSI images. To demonstrate the effectiveness of our approach, extensive experiments on six hyperspectral images, acquired by AVIRIS, HYDICE and HYPERION sensors, have been performed. The experimental results outcomes validate the effectiveness of the method in term of image quality. Indeed, the approach yields to high compression ratios with preserving most significant part of information in HSI images. Compared to other famous compression techniques such as the 3DSPECK, SSASR and 3D-DCT+SVM, the 3DDWT + SVR method substantially improves compression performances. Results obtained from applying 2DDWT for HSI data compression are more efficient than 3D-DWT in term of increasing distortion performance. In our case, we obtained for $\epsilon$ lower than 0.0001 with SVR gaussian kernel, an average PSNR close to 42.38 , 46.58, 46.10, $41.29 \mathrm{~dB}, 49.47 \mathrm{~dB}$ and $38.46 \mathrm{~dB}$ with bit rate about 0.7 for Yellowstone, Cuprite, Indiana, Washington DC, M.S.Helens and Erta Ale datasets respectively. Thus a PSNR improvement of at least 0.53 $\mathrm{dB}$ is observed. However, increasing compression performances does not necessarily improve specific data anal- 
ysis. Indeed to demonstrate the effectiveness of the proposed method in classification-based applications, we evaluate the classification performances. Results show that the method outperforms in terms of accuracy and McNemars's test. Furthermore, the spectral reflectance over bands of 3D-DWT + SVR compressed data show that the variations are almost identical to those of the original image. Finally we can highlight three major advantages of the proposed method: 1) from compression performances evaluation, the 3D-DWT + SVR substantially gives high compression ratios with good image quality 2) even with small training samples the compressed data gives high classification accuracy and outperforms those given by compared methods. In addition, method achieves better performances for compressed data than those of original data without compression 3) the proposed method presents spectral fidelity, which is substantially useful in specific-applications such as anomalies detection or data analysis. The major disadvantage of the method is the taken time execution, especially for high levels resolution which is mainly due to time processing of the SVR model. The use of parallel computation and $\mathrm{C} / \mathrm{C}++$ version codes are future prospects for improving the time execution of the method. Further work using Wavelet-Packet transform and CNN-based classifier for improving compression and classification performances is an interest perspective to consider.

\section{Compliance with Ethical Standards}

\subsection{Funding}

Not applicable

\subsection{Conflict of Interest}

The authors declare that they have no competing interests.

\section{References}

1. I., Prathap, R. Anitha, "Watermark Detection in Spatial and Transform Domains Based on Tree Structured Wavelet Transform," International Symposium on Security in Computing and Communication , Springer, Berlin, Heidelberg, pp. 230-238, September 2014.

2. B. Andries, J. Lemeire, A. Munteanu, "Scalable texture compression using the wavelet transform," The Visual Computer, vol. 33, no. 9, pp. 1121-1139, 2017.
3. P. Joshi, S. Prakash, S. Rawat, "Continuous wavelet transform-based no-reference quality assessment of deblocked images," The Visual Computer, vol. 34, no. 12, pp. 1739-1748, 2018.

4. L. He, Y. Wang, Z. Xiang, "Wavelet frame-based image restoration using sparsity, nonlocal, and support prior of frame coefficients," The Visual Computer, vol. 35, no. 2, pp. 151-174, 2019.

5. D. Venugopal, S. Mohan, S. Raja, "An efficient block based lossless compression of medical images" OptikInternational Journal for Light and Electron Optics, vol.127, no. 2, pp. 754-758, 2016.

6. Khiari-Hili, N., Lelandais, S., Montagne, C., Roumes, C., Hamrouni, K., Plantier, J.: Bio-inspired image enhancement derived from a rank order coding model," IET Image Processing, vol. 10, no. 5, pp. 409-417, 2016.

7. P. Govindan, J. Saniie, "Processing algorithms for three-dimensional data compression of ultrasonic radio frequency signals," IET Signal Processing, vol. 9, no.3, pp. 267-276, 2015.

8. M. S. Lee, S. K. Ueng, J. J. Lin, "Wavelets-based smoothness comparisons for volume data," IET Image Processing, vol. 9, no. 12, pp. 1057-1063, 2015.

9. K. J. Cheng, J. C. Dill, "An improved EZW hyperspectral image compression," Journal of Computer and Communications, vol. 2, no.02, pp. 31-36, 2014.

10. D. S. Sujithra, T. Manickam, D. S. Sudheer, "Compression of hyperspectral image using discrete wavelet transform and Walsh Hadamard transform," International Journal of Advanced Research in Electronics and Communication Engineering (IJARECE), vol. 2, pp. 314-319, 2013.

11. F. Aul í-Llin as, M. W. Marcellin, J. Serra-Sagrista, J.Bartrina-Rapesta, "Lossy-to-lossless 3D image coding through prior coefficient lookup tables", Information Sciences, vol. 239, pp. 266-282, 2013.

12. J. Delcourt, A. Mansouri, T. Sliwa, Y. Voisin, "An Evaluation Framework and a Benchmark for Multi/Hyperspectral Image Compression," Intelligent Computer Vision and Image Processing: Innovation, Application, and Design, pp. 56-66, 2013.

13. G. Hegde, P. Vaya, "Systolic array based motion estimation architecture of 3D DWT sub band component for video processing," International Journal of Signal and Imaging Systems Engineering, vol. 5, no. 3, pp. 158-166, 2012.

14. M. Lahdir, A. Nait-ali, S. Ameur, "Fast EncodingDecoding of 3D Hyperspectral Images Using a NonSupervised Multimodal Compression Scheme," Journal of Signal and Information Processing, vol. 2, no. 4, pp. 316-321, 2011. 
15. R. Jiao, Y. Li, Q. Wang, B. Li, "SVM regression and its application to image compression," Springer Berlin Heidelberg. Advances in Intelligent Computing, pp. 747-756, 2005.

16. Y. Li, H. Hu, "Image compression using wavelet support vector machines," International Conference on Intelligent Computing. Springer, Berlin, Heidelberg, pp. 922-929, August 2007.

17. .S. Fazli, S. Toofan, Z. Mehrara, " JPEG2000 Image Compression Using SVM and DWT," International Journal of Science and Engineering Investigations, vol. 1, pp. 53-57, 2012.

18. L. Zhang, L. Zhang, D. Tao, X. Huang, B. Du, "Compression of hyperspectral remote sensing images by tensor approach," Neurocomputing. vol. 147, pp. 358-363, 2015.

19. L. Fang, N. He, H. Lin, "CP tensor-based compression of hyperspectral images," Journal of the Optical Society of America A, vol. 34, no. 2, pp. 252-258, 2017.

20. B. Huang, Y. Nian, J. Wan, "Distributed lossless compression algorithm for hyperspectral images based on classification," Spectroscopy Letters, vol. 48, pp. 528-535, 2015.

21. N. Amrani, J. Serra-Sagrist , V. Laparra, M.W. Marcellin, J. Malo, "Regression Wavelet Analysis for Lossless Coding of Remote-Sensing Data," IEEE Trans. Geoscience and Remote Sensing. vol. 54, no. 9, pp. 5616-5627, September 2016.

22. Y. Meyer, "Wavelets and applications," Paris [etc.]: Masson, Berlin [etc.]: 'Springer-Verlag', 1992.

23. Y. Qian, M. Ye, "Hyperspectral imagery restoration using nonlocal spectral-spatial structured sparse representation with noise estimation," IEEE Journal of, Selected Topics in Applied Earth Observations and Remote Sensing, vol. 6, no. 2, pp. 499-515, 2013.

24. S. G. Mallat, "A theory for multiresolution signal decomposition: the wavelet representation," IEEE Trans. Pattern Analysis and Machine Intelligence, vol. 11, no.7, pp. 674-693, 1989.

25. J. M. Shapiro, "Embedded image coding using zerotrees of wavelet coefficients," IEEE Trans. Signal Processing., vol. 41, no. 12, pp. 3445-3462, 1993.

26. A. Said, W. A. Pearlman, "A new, fast, and efficient image codec based on set partitioning in hierarchical trees," IEEE Trans. Circuits and Systems for Video Technology, vol. 6, no. 3, pp. 243-250, 1996.

27. A. Islam, W. A. Pearlman, "Embedded and efficient low-complexity hierarchical image coder," International Society for Optics and Photonics. Electronic Imaging'99, pp. 294-305, 1998.

28. W. A. Pearlman, A. Islam, N. Nagaraj, A. Said," Low-complexity image coding with a set-partitioning embedded block coder," IEEE Trans. Circuits and Systems for Video Technology, vol. 14, no. 11, pp. 1219-1235, 2004.

29. D. Taubman, "High performance scalable image compression with EBCOT," IEEE trans. Image Process. vol. 9, no.7, pp.1158-1170, 2000.

30. S.G. Mallat, "Multifrequency channel decompositions of images and wavelet models," IEEE Trans. Acoustics, Speech and Signal Processing. vol. 37, no.12, pp. 2091-2110,1989.

31. B. J. Kim, W.A. Pearlman, "An embedded wavelet video coder using three-dimensional set partitioning in hierarchical trees (SPIHT)," Proc. Int. Conf. Data Compression Conference, DCC'97, IEEE, pp. 251260, 1997.

32. B. E. Reddy, K. V. Narayana, "A lossless image compression using traditional and lifting based wavelets," Signal and Image Processing. vol. 3, no. 2, pp. 213-222, 2012.

33. M. S. Abdullah, N.Subba Rao, " Image Compression using Classical and Lifting based Wavelets," International Journal of Advanced Research in Computer and Communication Engineering, vol. 2, no. 8, pp. 3193-3198, 2013.

34. Q. Wu, "A hybrid-forecasting model based on Gaussian support vector machine and chaotic particle swarm optimization," Expert Systems with Applications, vol. 37, no.3, pp. 2388-2394, 2010.

35. V. Vapnik, "The nature of statistical learning theory," in Springer Science Business Media, 2013.

36. J. Mercer, " Functions of positive and negative type, and their connection with the theory of integral equations," Philosophical transactions of the royal society of London, Series A, containing papers of a mathematical or physical character, vol.209, pp. 415-446, 1909.

37. P. G.Howard, J. S.Vitter, "Arithmetic Coding for Data Compression," Proc IEEE., vol. 82, no. 6, pp. 857-865, 1994.

38. Z. Wang, A. C. Bovik, H. R. Sheikh, E. P. Simoncelli, "Image quality assessment: from error visibility to structural similarity," IEEE Trans. Image Process., vol.13, no. 4, pp. 600-612, 2004.

39. Z. Wang, D. Zhang, and Y. Yu, "Video Quality Assessment based on Structural Distortion Measurement," Signal Processing: Image Communication, vol. 19, no. 2, pp. 121-132, Feb. 2004.

40. A. Villa, J.A. Benediktsson, J. Chanussot, C. Jutten, "Hyperspectral image classification with independent component discriminant analysis," IEEE Trans. Geosci. Remote Sens., vol. 49, pp. 4865-4876, 2011. 
41. L. Ran, Y. Zhang, W. Wei, Q. Zhang, "A hyperspectral image classification framework with spatial pixel pair features," Sensors, vol. 17, no. 10, pp. 2421, 2017.

42. W. Fu, S. Li, L. Fang, J. A. Benediktsson, "Adaptive Spectral-Spatial Compression of Hyperspectral Image With Sparse Representation," IEEE Transactions on Geoscience and Remote Sensing., pp. 1-12, 2016.

43. A. Karami, S. Beheshti, M. Yazdi, " Hyperspectral image compression using 3D discrete cosine transform and support vector machine learning," IEEE, Information Science Signal Processing and their Applications (ISSPA), pp. 809-812, 2012.

44. X. Tang and W. A. Pearlman, "Three-dimensional wavelet-based compression of hyperspectral images," in Hyperspectral Data Compression, New York, NY, USA: Springer-Verlag, 2006, ch. 10, pp. 273-278.

45. Q. Du and J. E. Fowler, "Hyperspectral image compression using JPEG2000 and principal component analysis," IEEE Geosci. and Remote Sens. Lett., vol. 4, pp. 201-205, Apr. 2007.

46. Q. Du, N. Ly, J. E. Fowler, "An operational approach to PCA+JPEG2000 compression of hyperspectral imagery," IEEE J. Sel. Topics Appl. Earth Observ. Remote Sensing., vol. 7, no. 6, pp. 2237-2245, Jun. 2014.

47. F. Garcia-Vilchez et al., "On the impact of lossy compression on hyperspectral image classification and unmixing," IEEE Geosci. Remote Sensing. Lett., vol. 8, no. 2, pp. 253-257, Mar. 2011.

48. S. Boussakta and H. O. Alshibami, "Fast Algorithm for the 3-D DCT-II," IEEE TRANS. ON Signal Processing, vol. 52, pp. 992-1001, Apr. 2004.

49. A. Karami, M. Yazdi, G. Mercier, "Compression of hyperspectral images using discerete wavelet transform and tucker decomposition," IEEE J, Sel. Topics Appl. Earth Observ. Remote Sensing., vol 5, no 2, pp. 444-450, Apr. 2012.

50. N. Zikiou, M. Lahdir, S. Ameur, "Color image compression based on wavelet transform and support vector regression," Image Processing, Applications and Systems Conference (IPAS), 2014 First International IEEE, pp. 1-6, Nov. 2014.

51. W. Hu, Y. Huang, W. Li, F. Zhang, H. Li, "Deep convolutional neural networks for hyperspectral image classification," J. Sensors, vol. 2015, Jan. 2015, Art. no. 258619, 2015.

52. L. Mou, P. Ghamisi, X.X. Zhu, "Deep recurrent neural networks for hyperspectral image classification," IEEE Trans. Geosci. Remote Sens., vol. 55, no.7, pp. 3639-3655, 2017.
53. W. Li, G. Wu, F. Zhang, Q. Du, "Hyperspectral image classification using deep pixel-pair features," IEEE Transactions on Geoscience and Remote Sensing, vol. 55, no. 2, pp. 844-853, 2017.

54. Z. Li. J.Zhong, Z. Luo, M. Chapman, " SpectralSpatial Residual Network for Hyperspectral Image Classification: A 3-D Deep Learning Framework," IEEE Transactions on Geoscience and Remote Sensing, vol. 56, no. 2, pp. 847-858, 2018.

55. M. Li. W. Zhang, Q. Du, "Diverse Region-Based CNN for Hyperspectral Image Classification," IEEE Transactions on Image Processing, vol. 27, no. 6, pp. 2623-2634, 2018.

56. H. Lee, H. Kwon, "Going deeper with contextual CNN for hyperspectral image classification," IEEE Trans, Image Process., vol. 26, no. 10, pp. 4843-4855, Oct. 2017. 